\title{
Multiple-Input Multiple-Output (MIMO) linear systems extreme inputs/outputs
}

\author{
David O. Smallwood* \\ 9817 Pitt Pl. NE, Albuquerque, NM 87111, USA
}

Received 29 September 2005

Revised 15 March 2006

\begin{abstract}
A linear structure is excited at multiple points with a stationary normal random process. The response of the structure is measured at multiple outputs. If the autospectral densities of the inputs are specified, the phase relationships between the inputs are derived that will minimize or maximize the trace of the autospectral density matrix of the outputs. If the autospectral densities of the outputs are specified, the phase relationships between the outputs that will minimize or maximize the trace of the input autospectral density matrix are derived. It is shown that other phase relationships and ordinary coherence less than one will result in a trace intermediate between these extremes. Least favorable response and some classes of critical response are special cases of the development. It is shown that the derivation for stationary random waveforms can also be applied to nonstationary random, transients, and deterministic waveforms.
\end{abstract}

\section{Introduction}

The application of multiple inputs, in both test and analysis, to excite a single structure is becoming more common. In general a stationary random excitation of this nature requires the specification of the complete cross spectral density matrix of the inputs. If the inputs are transients the specification of the complex Fourier spectra is required. However, many times little is known about the exact nature of the inputs. Often, the only known information is the desired autospectra (or Fourier magnitude for transients) at the inputs or at the response points.

For example, the response of a structure at one or several points has been measured in field experiments and the desire is to replicate the response in a laboratory test. Or in the case of analysis, to excite a model of the system that will replicate the field measured response. In general there are multiple solutions to the problem. This paper will explore a specific class of solutions.

In other cases the autospectral densities at several inputs are specified. The inputs can be measured experimental inputs or envelopes derived from a variety of sources. Inconsistencies in these specifications can arise from a variety of sources. The source could be noisy. The source could be a composite of several experiments. The test item or model may differ from the system from which the source was derived resulting in shifting of modal frequencies and mode shapes. In any of these cases the specification of the phase relationships between the inputs is difficult or impossible. Certain phase relations may result in unrealistic inputs or responses. These inputs in essence impose boundary conditions on the model or experiment to force the desired response.

A simple example is a rod excited in the axial direction with forces at the two ends. Intuition tells us that the bar must move as a rigid body at very low frequencies. If the required motion at two response points is not identical, large input forces can result, because higher modes must also be excited to achieve the desired response. As the frequency increases excitation of the higher modes becomes easier and the achievement of different motion at two points becomes easier as a combination of the rigid body translation and higher modes. At low frequencies the rigid

\footnotetext{
*Tel.: +1 505296 2931; Fax: +1 505296 2931; E-mail: dosmallwood@ comcast.net.
} 


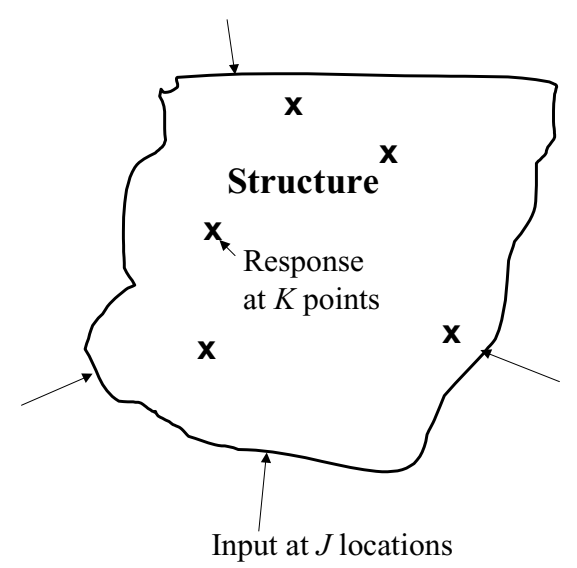

Fig. 1. General multiple-input, multiple-output (MIMO) linear system.

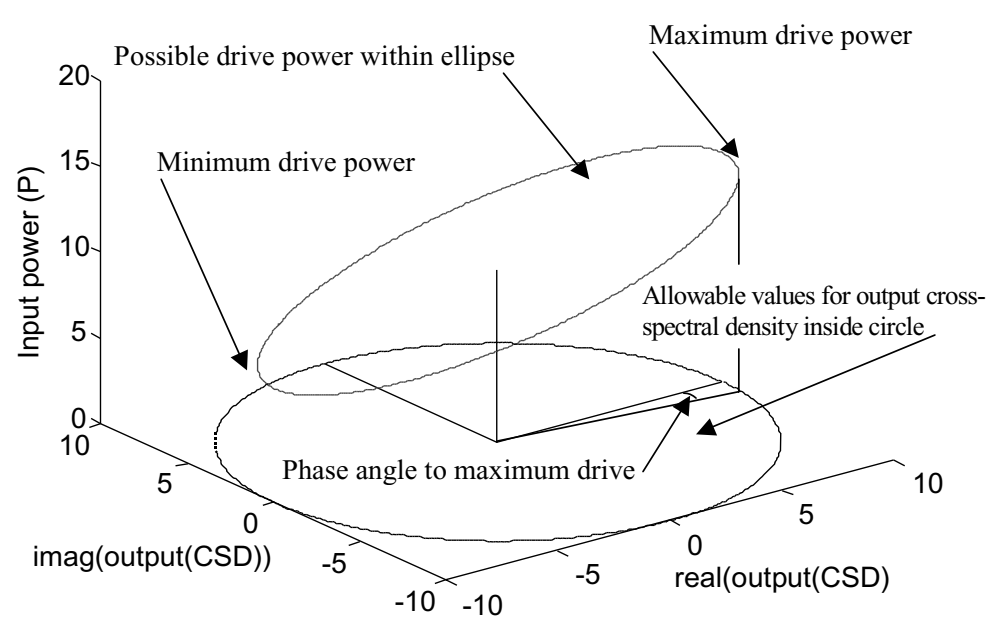

Fig. 2. Geometric interpretation of input requirement.

body mode is excited most efficiently with the forces at the end in phase. Near the first longitudinal mode the bar is excited most efficiently with forces at the two ends out of phase. The forces required to achieve a given response is influenced by the crossover frequency where the excitation is changed from in-phase to out-of-phase.

This paper attempts to find a rational solution to minimize the forces required to achieve a specified response. For the case of specified response autospectra density, the solution suggested is the solution that minimizes the trace of the input autospectral density matrix. The solution that maximizes the trace of the input autospectra is also derived. The minimum trace solution is a reasonable excitation because it excites the structure in a manner consistent with the dynamic properties of the structure and does not require unreasonable boundary conditions for the structure under test or analysis. It is shown that any other solution will result in a trace between the minimum and maximum solution. The problem has been solved for a square system (the number of inputs equals the number of response points) [12]. This paper will generalize the results and extends the results to rectangular systems.

The case of the transient input with a single input and a single output (SISO) has been solved $[3,8]$ and is called the least favorable response. Other cases with single inputs and responses have also been studied are called the critical response $[1,4,5,14]$.

In the examples of this paper the input is force and the output is acceleration. But it is important to understand that the method is much more general. For example, the input could be voltage into a power amplifier driving a shaker. The output could be stress or strain. Any pair of quantities for which linear input/output relationships can be derived or measured can be used. 
Input and response
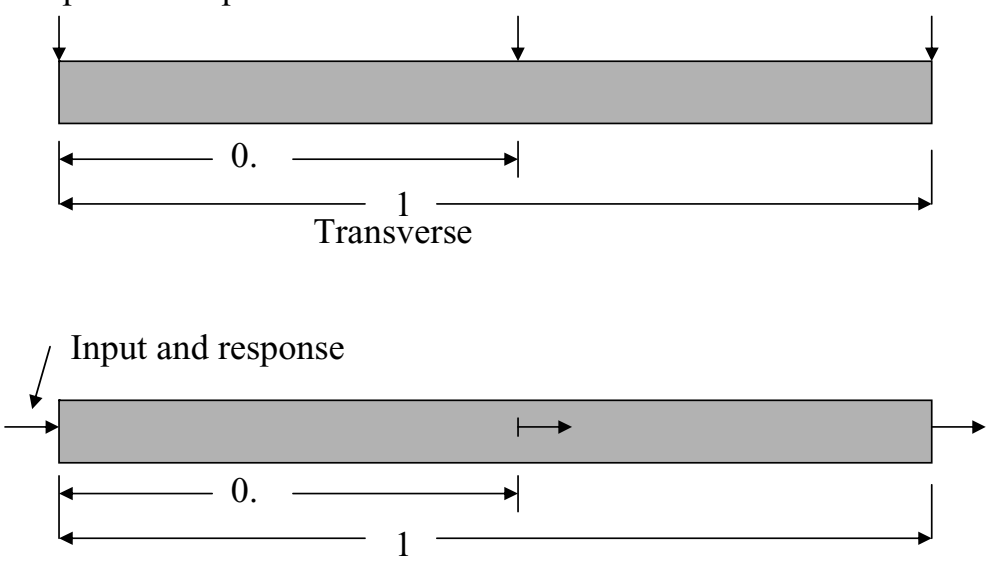

Longitudinal

Fig. 3. Sketch of bars used in examples.
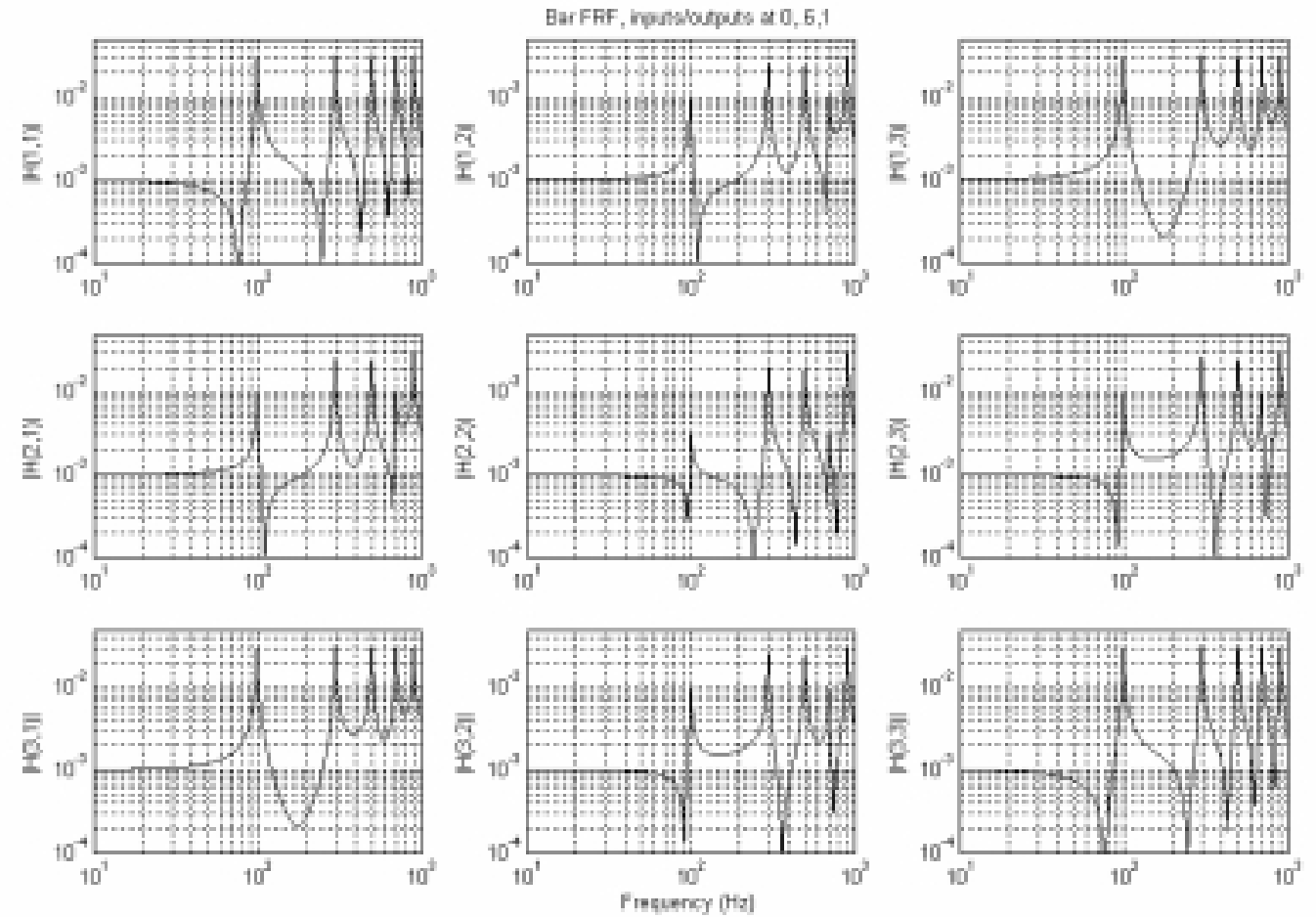

Fig. 4. FRF's, bar, magnitude $(\mathrm{g} / \mathrm{N})$.

Several related problems involving a multiple-input, multiple-output (MIMO) system are discussed in this paper. In general, these problems fall into three classes: 1) the number of inputs exceeds the number of outputs, 2) the number of inputs is the same as the number of inputs, and 3) the number of outputs exceeds the number of inputs. All are discussed.

The first class of problems discussed is where the autospectral densities of the outputs are specified, but the output 

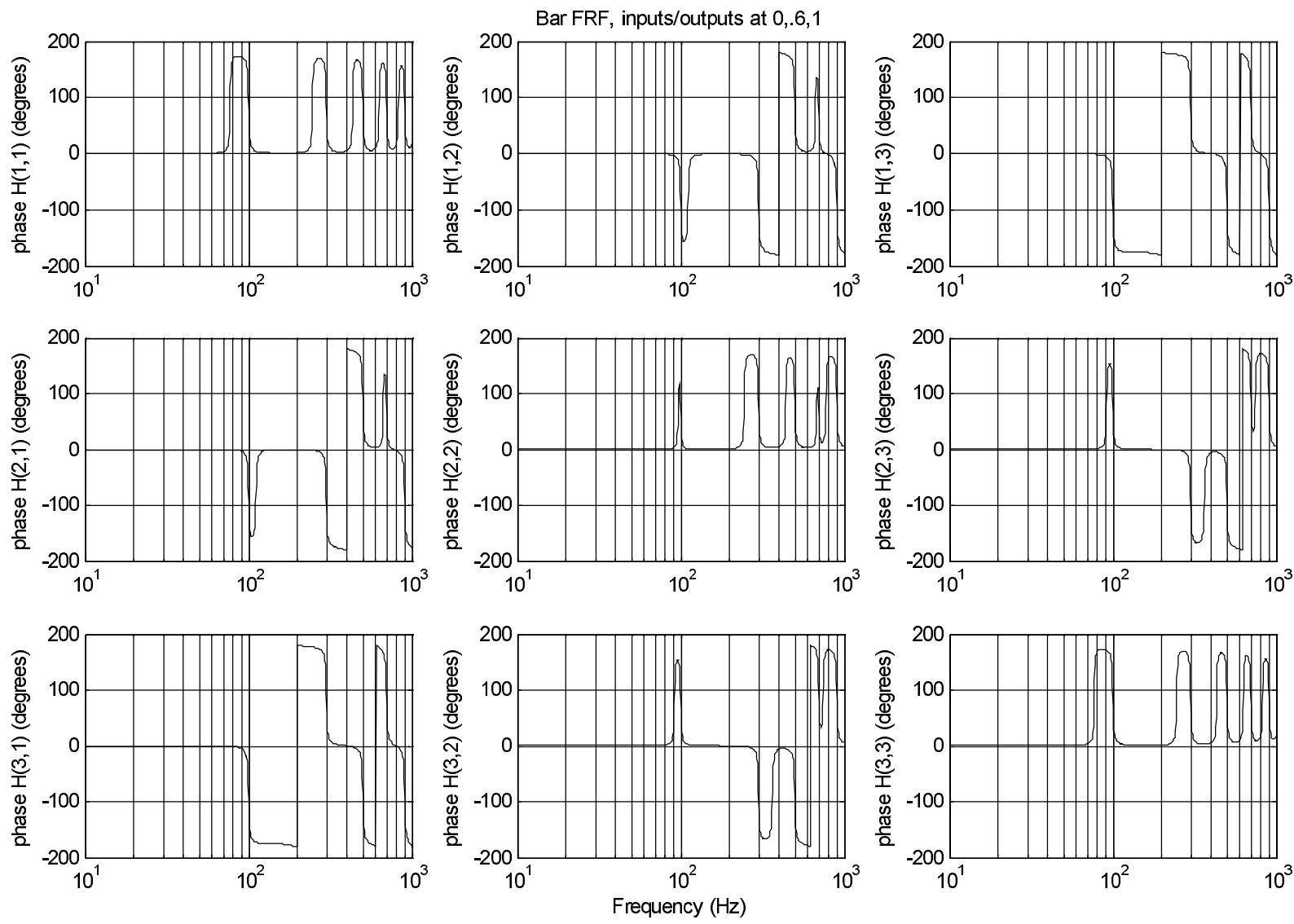

Fig. 5. FRF's, $1000 \mathrm{lb}$ bar, phase (degrees).

cross spectral densities are free variables. The problem is to derive a set of inputs that will produce the required output autospectral densities. The solution is not unique. The solution derived for the output cross spectral densities minimizes (or maximizes) the sum of the input autospectral densities (the trace of the input cross spectral density matrix). Other solutions are shown to be intermediate to these two extremes. A geometric interpretation of the solution is given.

The second related class of problems is where the input autospectral densities are specified, but where the input cross spectral densities are free variables. Similar to the first problem, the solution derived generates the input cross spectral densities that will minimize (or maximize) the sum of the output autospectral densities.

It is then shown how the random solution can be applied to other related problems: 1) a transient input, 2) the method of least favorable (or critical response), 3) a pseudo random input, 4) a deterministic input.

Two special cases of a multiple input single output (MISO) systems are then discussed. The first is the case where the single output is a quadratic combination of linear outputs that can be decomposed into a linear MIMO system using Cholesky decomposition. Von Mises stress is an example of such a single output. The other case discussed is a method to evaluate the equivalence between three single axis vibration tests and a single multiple input vibration test.

The last section includes five examples to illustrate the solutions derived.

\section{Derivation of the minimum/maximum input trace}

As a practical matter most of the derivations are in the frequency domain. It is assumed that the inputs and responses are sampled at equal time intervals and the random spectra are specified at finite number of evenly spaced 

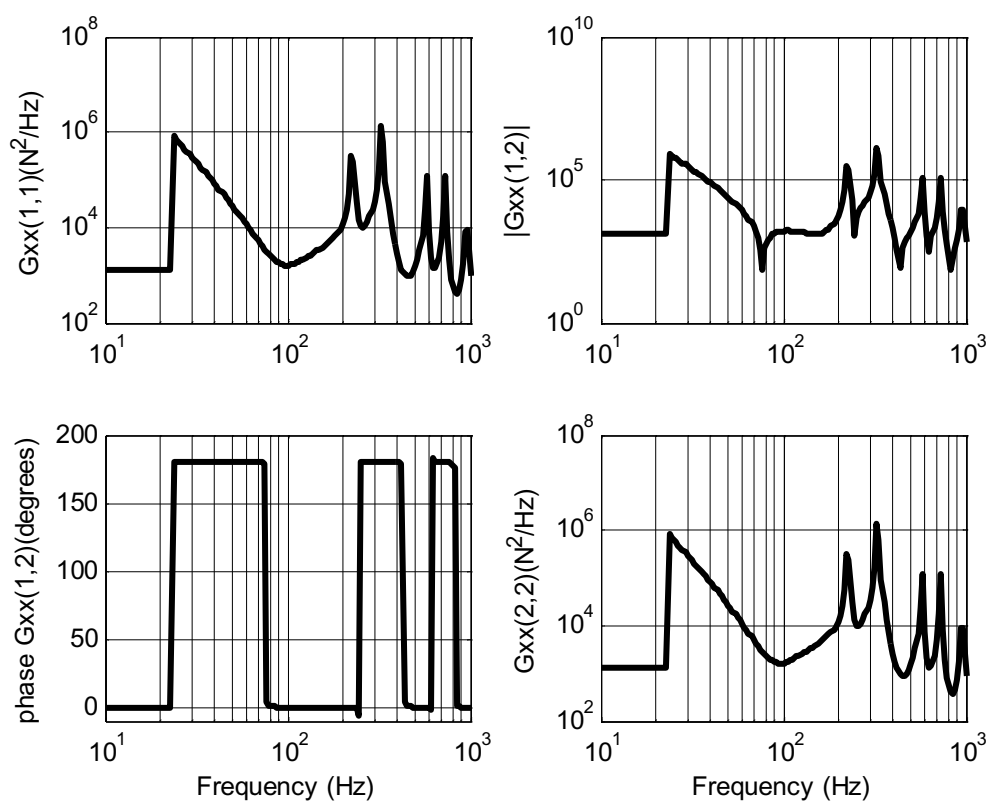

Fig. 6. Example 1, Input cross spectral density.
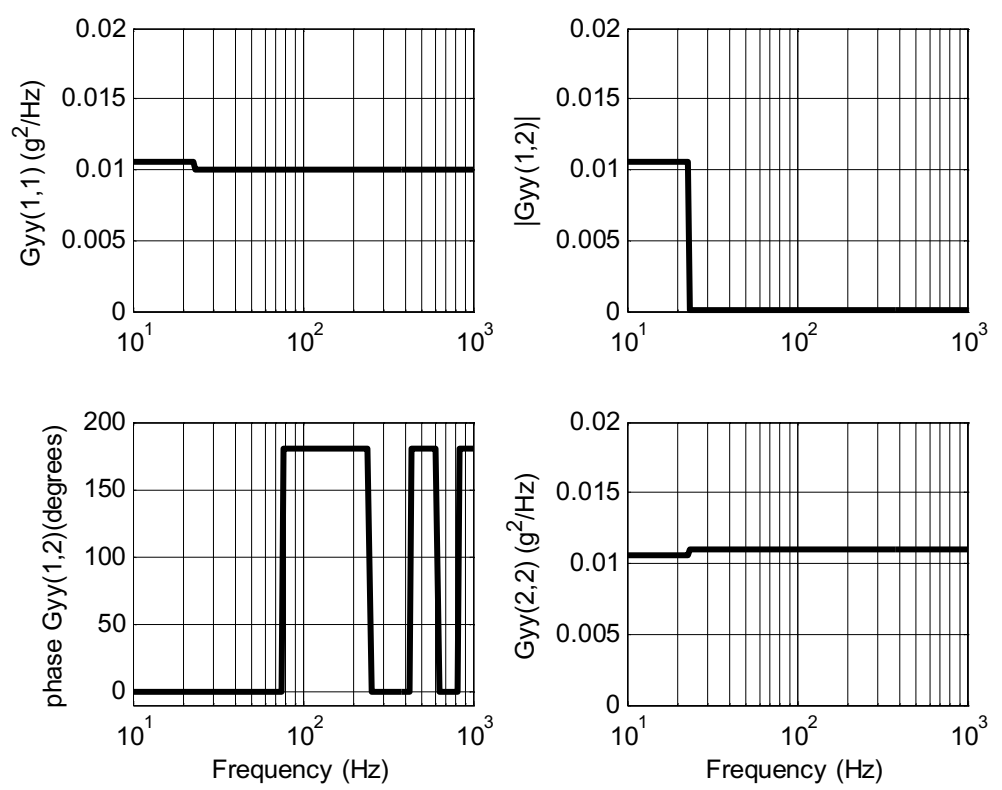

Fig. 7. Example 1, Output spectral density.

frequency lines between zero and half the sampling frequency (the Nyquist frequency). For transients, the spectra are specified using the discrete Fourier transform as an approximation of the continuous transform.

A general multiple input multiple output (MIMO) linear system is represented in Fig. 1.

The inputs can be represented as a vector and the outputs as another vector. Each element in the vectors is a time history. The elements in the vectors can also be represented in the frequency domain by the Fourier transform.

$$
X(f)=\int_{-\infty}^{\infty} x(t) e^{-i 2 \pi f t} d f
$$




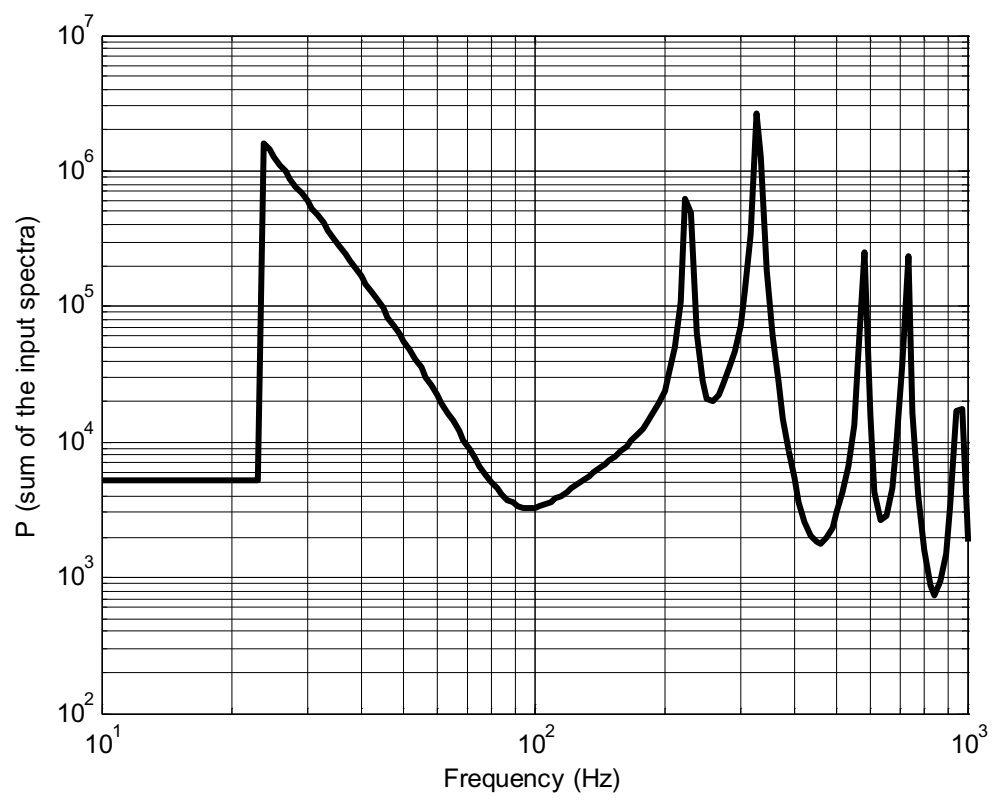

Fig. 8. Example 1, Trace of the input spectral density.

This integral does not strictly exist for a stationary random signal and a limiting operation must be defined. To simplify the notation let

$$
\mathbf{S}_{\mathbf{X X}}=E\left[\mathbf{X X}^{\prime}\right]
$$

where $\mathbf{X}$ is a column vector of the discrete Fourier transforms of a realization of a column vector of the time histories, $\mathbf{x}$. Justification for this simplification is contained in [2]. Let $\mathbf{H}$ represent a matrix of frequency response functions relating the inputs to the outputs of a MIMO system. The cross spectral density matrix of the outputs, $\mathbf{S}_{\mathbf{Y Y}}$, can be stated in terms of the cross spectral density matrix of the inputs, $\mathbf{S}_{\mathbf{X X}}$, as

$$
\mathbf{S}_{\mathbf{Y Y}}=\mathbf{H S}_{\mathbf{X X}} \mathbf{H}^{\prime}
$$

Define $\mathbf{G}$ as the Moore-Penrose pseudo inverse of $\mathbf{H}$

$$
\mathbf{G}=\operatorname{pinv}(\mathbf{H})
$$

If $\mathbf{H}$ is square and non-singular the pseudo inverse is the same as the matrix inverse. For the cases where $\mathbf{H}$ is rectangular or singular the Moore-Penrose pseudo inverse produces the result

$$
\begin{aligned}
& \mathbf{H G H}=\mathbf{H} \\
& \mathbf{G H G}=\mathbf{G}
\end{aligned}
$$

GH and HG are Hermitian.

Let norm $(\mathbf{H})$ be defined as the largest singular value of $\mathbf{H}$. If $\mathbf{H}$ has more rows than columns (more outputs than inputs) or is square and singular then the inverse of $\mathbf{H}$ does not exist. In these cases pinv (H) has some, but not all, of the properties of the inverse of $\mathbf{H}$. If $\mathbf{H}$ has more rows than columns and is not of full rank, then the overdetermined least squares problem

$$
\text { minimize } \operatorname{norm}(\mathbf{H X}-\mathbf{Y})
$$

does not have a unique solution. The solution

$$
\mathbf{X}=\operatorname{pinv}(\mathbf{H}) \mathbf{Y}
$$

is the solution for which the $\operatorname{norm}(\mathbf{X})$ is the smallest. 

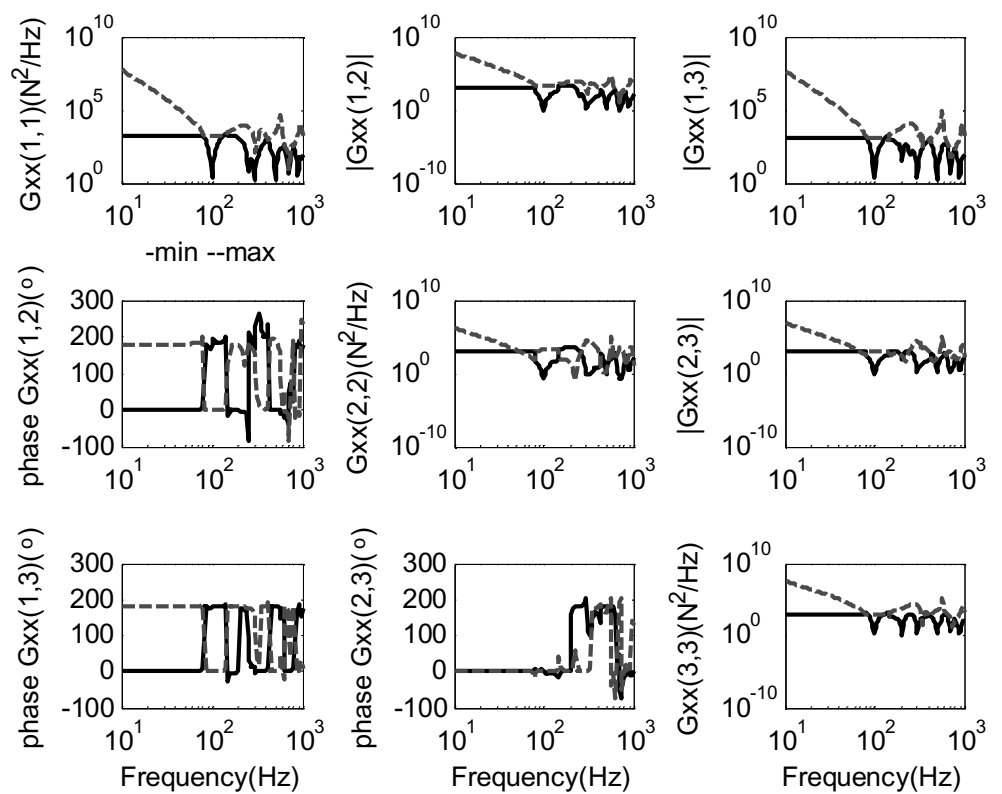

Fig. 9. Example 2, Input spectral density.
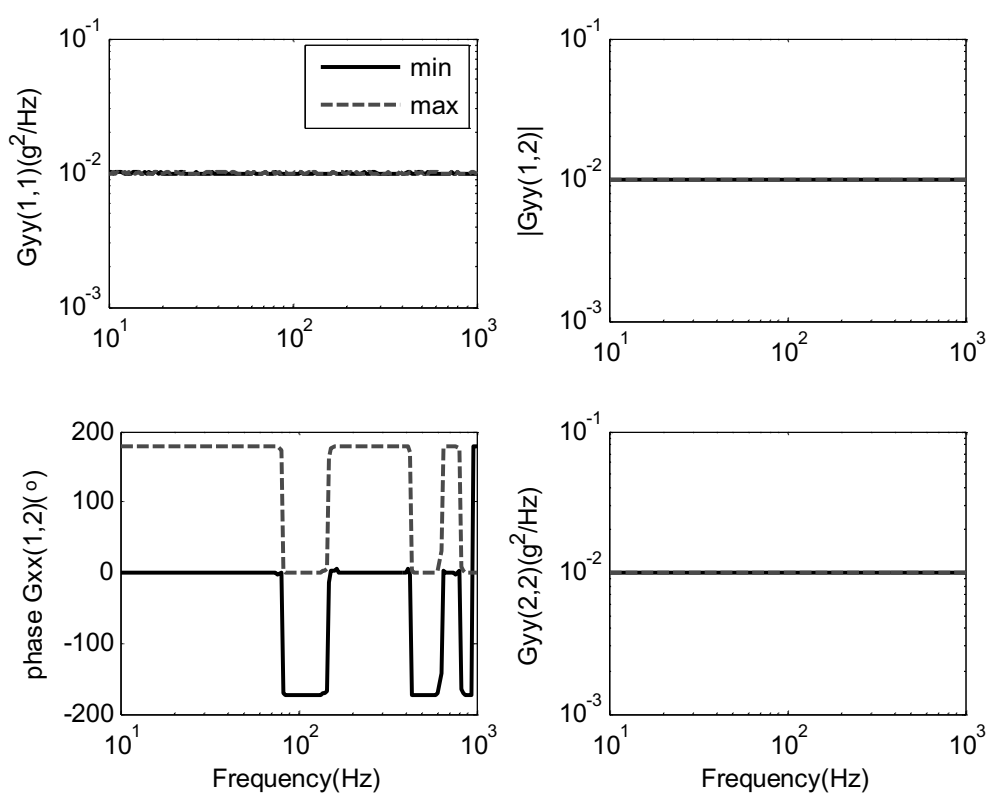

Fig. 10. Example 2, Output spectral density.

If $\mathbf{H}$ has more columns than rows (the number of inputs exceeds the number of outputs) there are many solutions to the problem

$$
\mathbf{H X}-\mathbf{Y}=0
$$

The solution of the off-diagonal terms of $\mathbf{S}_{\mathbf{Y Y}}$ that minimizes the trace of $\mathbf{S}_{\mathbf{X X}}$ when the diagonal elements of $\mathbf{S}_{\mathbf{Y Y}}$ are specified will be derived. The case where $\mathbf{H}$ is square and full rank is a special case of this problem. In this case $\mathbf{G}$ is just the inverse of $\mathbf{H}$. 


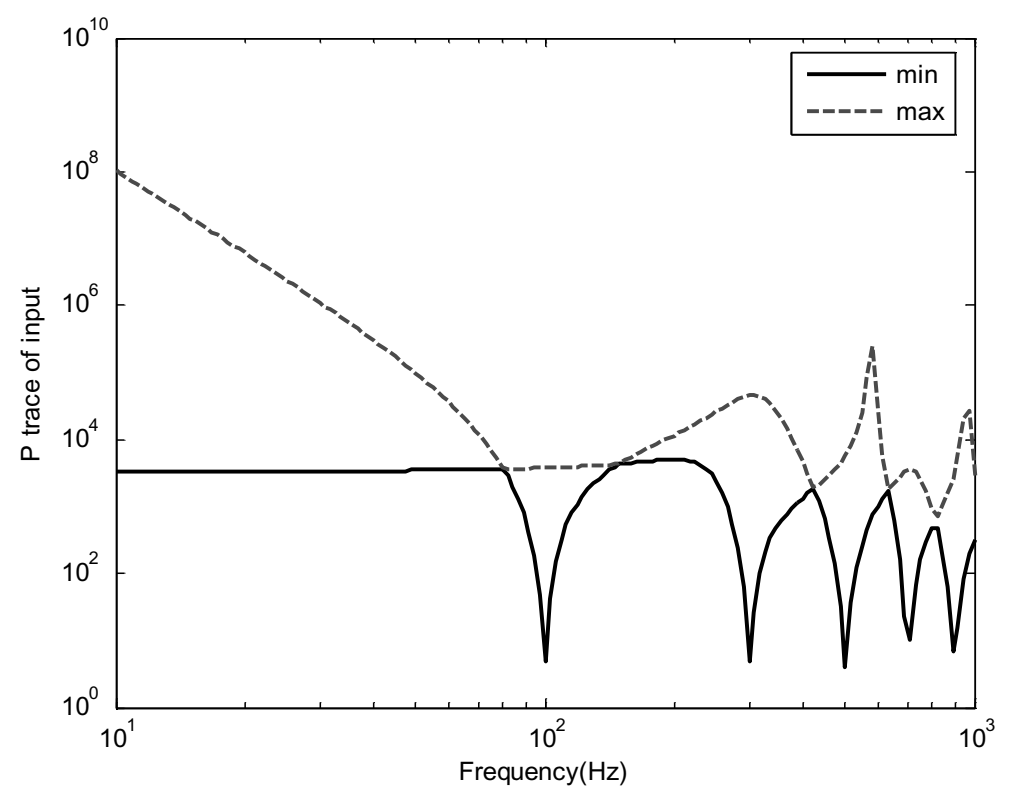

Fig. 11. Example 2, Trace of input spectral density.

The spectral density matrix of the input can now be expressed in terms of the spectral density matrix of the output as

$$
\mathbf{S}_{\mathbf{X X}}=\mathbf{G S}_{\mathbf{Y Y}} \mathbf{G}^{\prime}
$$

As a side note: If the complete cross spectral density matrix of the output is specified (not just the diagonal autospectra), Eq. (9) is used to compute the required input cross spectral density matrix.

Define a measure of the spectral input to the system as the sum of the autospectra of the inputs

$$
P=\operatorname{trace}\left(\mathbf{S}_{\mathbf{X X}}\right)
$$

This will be called the input power. It is not physical power except in special cases. One of the special cases comes from transmission theory using constant real impedance transmission lines, and hence the name). Using Eqs (9) and (10)

$$
\begin{aligned}
& P=\operatorname{trace}\left(\mathbf{G S}_{\mathbf{Y} \mathbf{Y}} \mathbf{G}^{\prime}\right) \\
& P=\sum_{j=1}^{J} \sum_{k=1}^{K} \sum_{m=1}^{K} G_{j k Y Y} S_{k m} G_{j m}^{*}
\end{aligned}
$$

where ${ }_{Y Y} S_{k m}$ is and element from $S_{Y Y}, J$ is the number of inputs, and $K$ is the number of outputs. It is important to note that ${ }_{Y Y} S_{k m}$ appears in the above equation only as a linear term. Cross products of ${ }_{Y Y} S_{k m}$ do not appear. This implies that the surface of $P$ above each complex ${ }_{Y Y} S_{k m}$ is a plane with a constant gradient. If we specify the autospectra of the outputs, ${ }_{Y Y} S_{k k}$, the range of ${ }_{Y Y} S_{k m}$ is restricted to

$$
0 \leqslant\left.||_{Y Y} S_{k m}\right|^{2} \leqslant{ }_{Y Y} S_{k k Y Y} S_{m m}
$$

The gradient of $P$ is constant, therefore the minimum and maximum will occur on the boundary of the range of the cross-spectrum. Ignoring the trivial result $\left(\left.||_{Y Y} S_{k m}\right|^{2}=0\right)$ set

$$
{ }_{Y Y} \bar{S}_{k m}=\left|{ }_{Y Y} S_{k m}\right|=\sqrt{Y Y S_{k k Y Y} S_{m m}}
$$

Interchanging the order of summation in Eq. (11) 

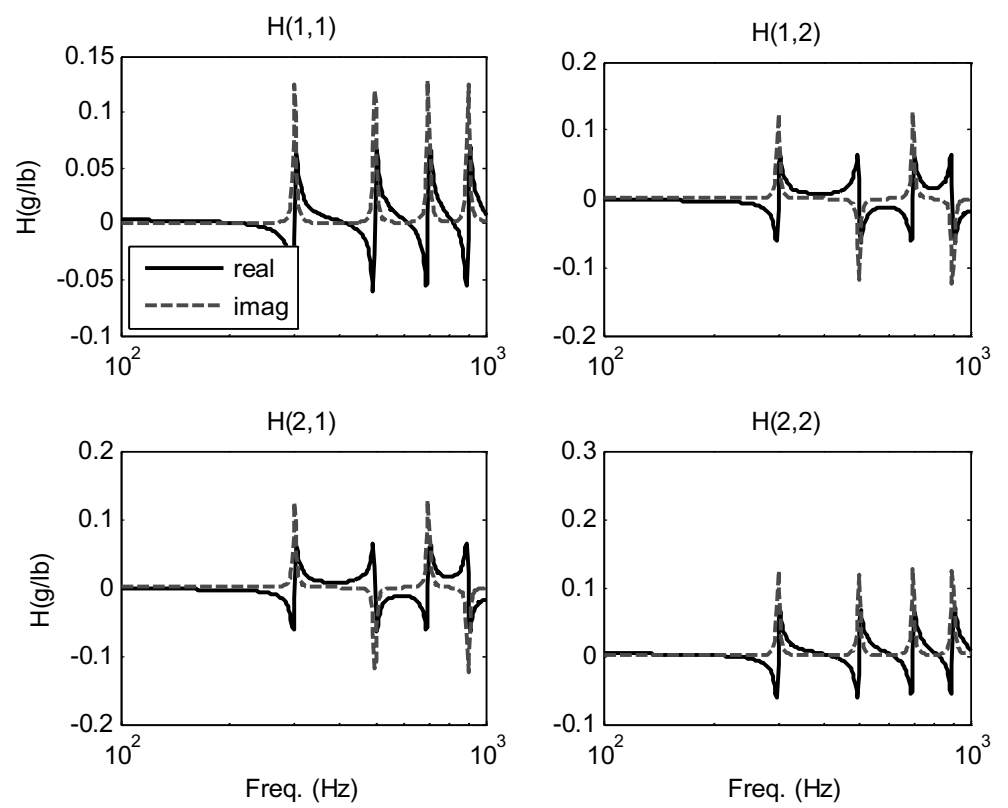

Fig. 12. Example 3, Beam frequency response functions.

$$
P=\sum_{k=1}^{K} \sum_{m=1}^{K} Y Y S_{k m} \sum_{j=1}^{J} G_{j m}^{*} G_{j k}
$$

Let

$$
\begin{aligned}
& \mathbf{F}=\mathbf{G}^{\prime} \mathbf{G} \\
& \text { or } \\
& F_{m k}=\sum_{j=1}^{J} G_{j m}^{*} G_{j k} \quad m=1 \ldots K, \quad k=1 \ldots K
\end{aligned}
$$

Substituting Eq. (15) into Eq. (14) gives

$$
P=\sum_{k=1}^{K} \sum_{m=1}^{K} Y Y S_{k m} F_{m k}
$$

Using the fact that both $\mathbf{S}_{Y Y}$ and $\mathbf{F}$ are Hermitian Eq. (16) can be expressed as

$$
P=\sum_{k=1}^{K} Y_{Y} S_{k k} F_{k k}+2 \sum_{k=1}^{K-1} \sum_{j=k+1}^{K} \operatorname{Re}\left({ }_{Y Y} S_{k j} F_{k j}^{*}\right)
$$

This expression can be written as

$$
P=\sum_{k=1}^{K} Y Y S_{k k} F_{k k}+2 \sum_{k=1}^{K-1} \sum_{j=k+1}^{K} Y Y \bar{S}_{k j} \bar{F}_{k j} \cos \left(a_{k j}-b_{k j}\right)
$$

where

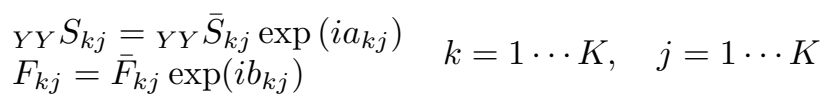

$P$ will be a minimum when all the terms in the second double sum of Eq. (18) are negative. The maximum will occur when all the terms are positive. The minimum will occur when 

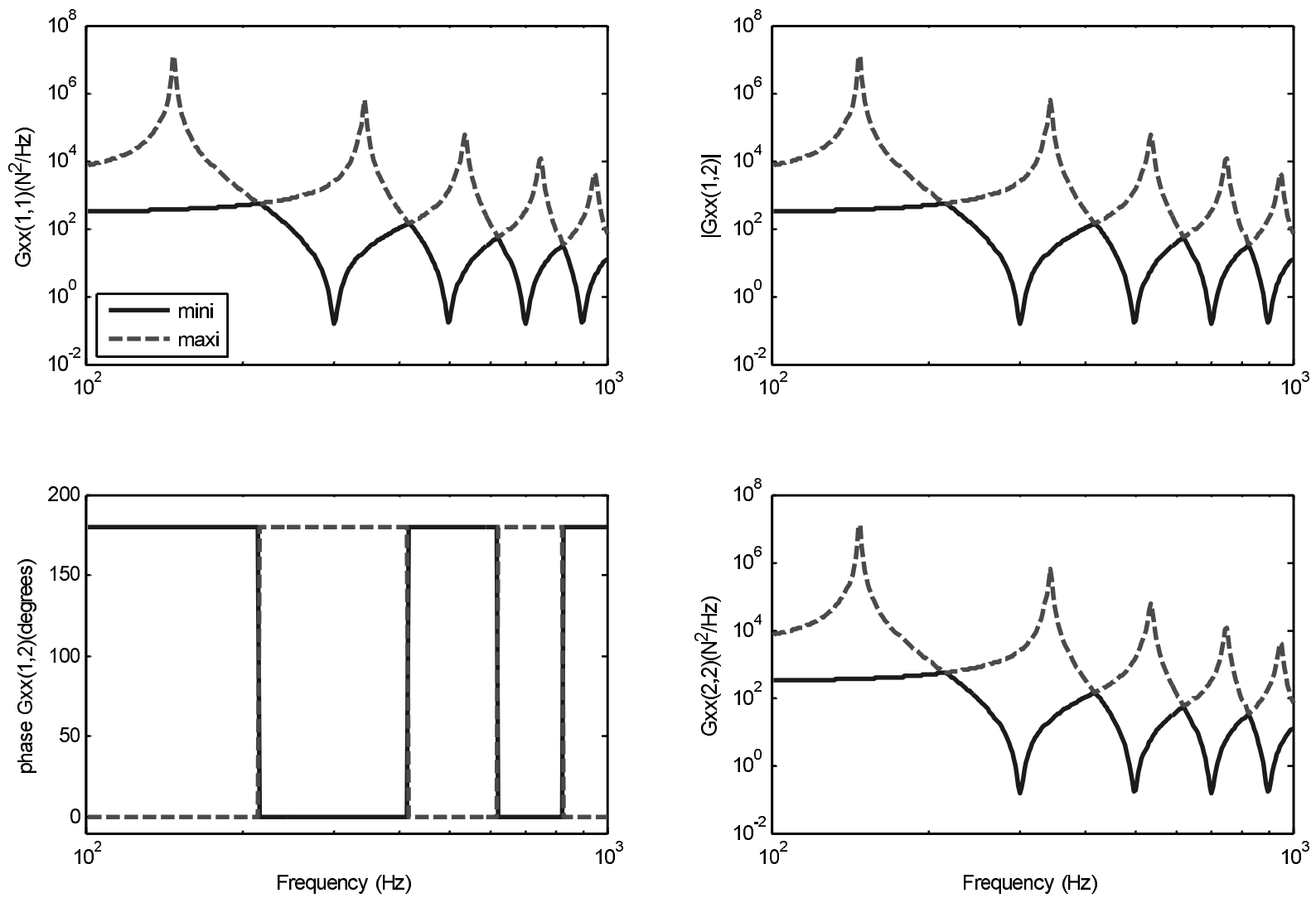

Fig. 13. Example 3, Input cross spectral density matrix.

$a_{j k}-b_{j k}=\pi \quad k=1 \cdots K, \quad j=1 \cdots K$

or

$$
\begin{aligned}
& a_{j k}=\pi+b_{j k} \quad k=1 \cdots K, \quad j=1 \cdots K \\
& { }_{Y Y} \widehat{S}_{j k}={ }_{Y Y} \bar{S}_{j k} \exp \left(i\left(b_{j k}+\pi\right)\right) \quad k=1 \cdots K, \quad j=1 \cdots K
\end{aligned}
$$

The maximum will occur when

$$
a_{j k}=b_{j k}
$$

The minimum $P$ is given by

$$
P=\sum_{k=1}^{K} Y Y S_{j j} F_{j j}-2 \sum_{k=1}^{K-1} \sum_{j=k+1}^{K} Y Y \bar{S}_{k j} \bar{F}_{k j}
$$

The maximum is given by

$$
P=\sum_{k=1}^{K} Y Y S_{k k} F_{k k}+2 \sum_{k=1}^{K-1} \sum_{j=k+1}^{K} Y Y \bar{S}_{k j} \bar{F}_{k j}
$$

To summarize, when the output autospectra are specified, the input with the minimum trace is given by

$$
\mathbf{S}_{\mathbf{X X}}=\mathrm{GS}_{\mathbf{Y} Y} \mathbf{G}^{\prime}
$$



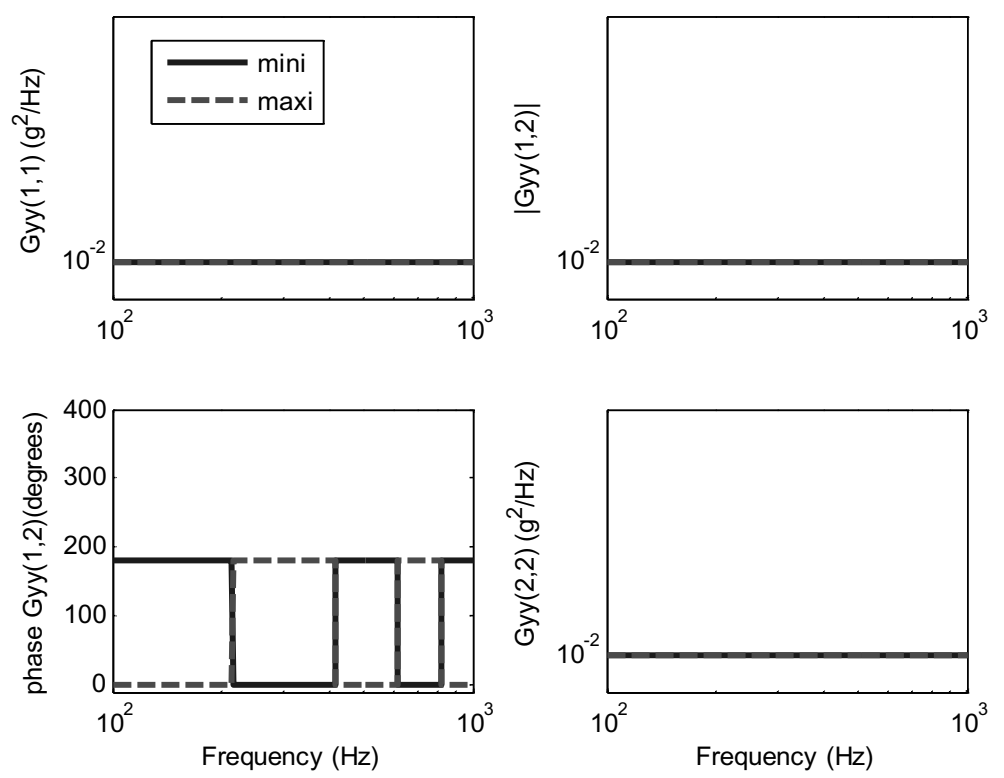

Fig. 14. Example 3, Output spectral density matrix.

where $\widehat{\mathbf{S}}_{\mathbf{Y Y}}$ is specified by Eqs (19) and (21). The response to this input is given by

$$
\mathrm{S}_{\mathbf{Y Y}}=\mathrm{HS}_{\mathbf{X X}} \mathbf{H}^{\prime}=\mathbf{H G S}_{\mathbf{Y} Y} \mathbf{G}^{\prime} \mathbf{H}^{\prime}
$$

The individual input pairs are fully coherent, which means the vector input can be specified with a single random noise source

$$
\mathbf{S}_{\mathbf{X X}}=\mathbf{X W \mathbf { X } ^ { \prime }}
$$

where $\mathbf{X}$ is now a deterministic complex column vector that is a function of frequency. $\mathbf{W}$ is the auto spectrum of a white noise source with a unity spectral density. $\mathbf{W}$ is a real scalar function of frequency (i.e. $\mathbf{W}=1$ ), which could be factored out. However to keep Eq. (28) as the same form as Eq. (26) this will not be done.

When the number of inputs exceeds the number of outputs the maximum input has a special meaning. It is not the maximum input that could be derived, which is speculated to be unbounded. It is the maximum input that can be achieved within the constraints of the pseudo inverse, which minimizes the trace of the input spectral density matrix.

For the special case of a single output, $\mathbf{F}$ (as defined in Eq. 15) is a real scalar and the maximum and minimum inputs are the same. The pseudo inverse will result in the input spectral density matrix with the minimum trace.

Underwood and Keller [15] used this result to generate inputs to a multiple shaker control system where the control was a linear combination of several measurement channels. In this method the matrix defining the linear combination of measurements is combined with $\mathbf{H}$ to generate a new transfer function. The pseudo inverse of the combined transfer function will result in the input spectral density matrix with the minimum trace.

\subsection{Geometric interpretation of input requirement}

For simplicity, first consider the case of two inputs and two outputs at one frequency. The autospectral densities of the two outputs are specified and we want to find the input autospectral densities for all possible values of the cross spectrum between the outputs. In this example, the magnitude of the autospectral densities of the two outputs is assumed to be the same. We will construct a three-dimensional plot where the first axis represents the real part of the cross spectral density, the second axis represents the imaginary part of the cross spectral density, and the third axis represents the power ( $P$, the sum of the two input auto (power) spectral densities, the trace of the input spectral density matrix). The allowable output spectra are represented by the interior of a circle on the x-y plane. Points on the circle represent unity coherence of the outputs with the varying phase of the cross-spectral density. Points in the 


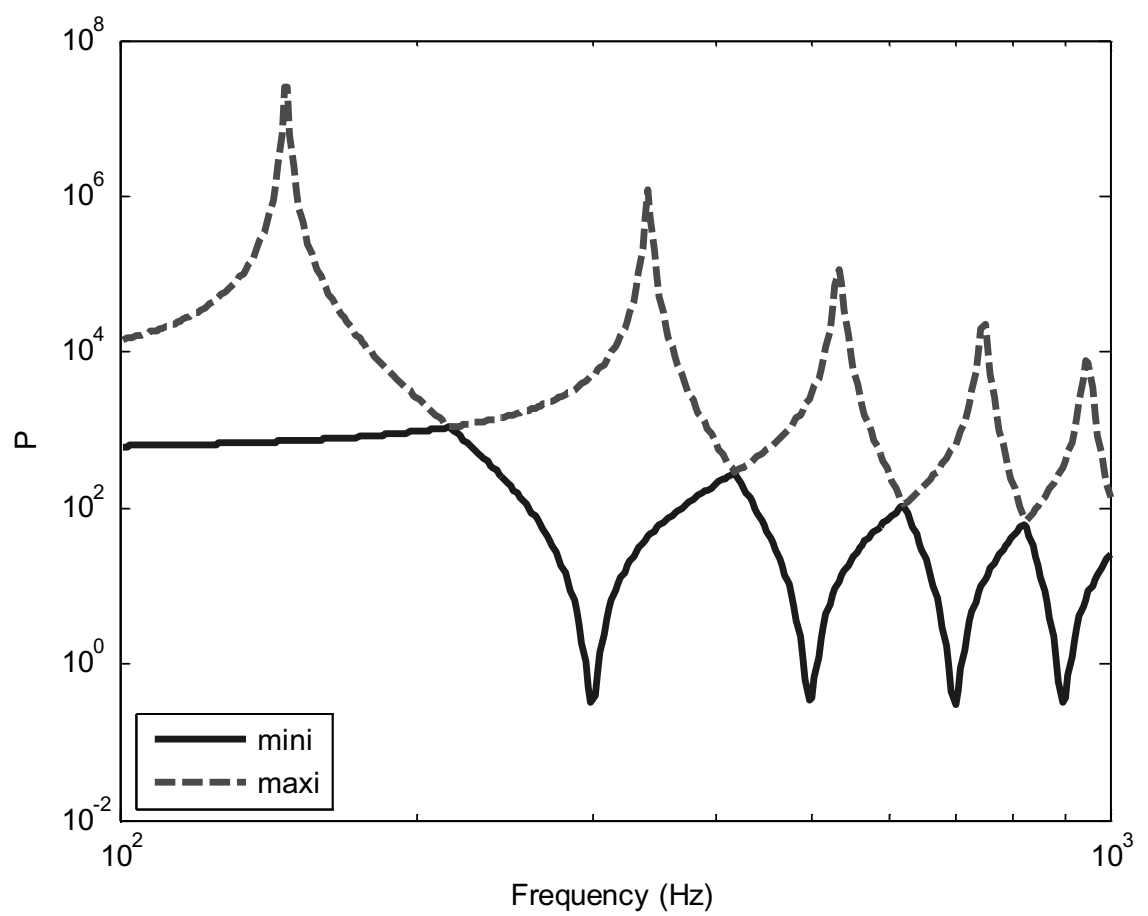

Fig. 15. Example 3, Trace of the input cross spectral density matrix.

interior of the circle will represent cross spectral densities with less than unity coherence. A point at the center of the circle will represent zero coherence between the two outputs. The gradient of $P$ is constant, and is therefore a tilted plane above the $x-y$ plane. The circle of allowable outputs is projected as an ellipse on the power plane. Figure 2 illustrates the plot. Points on the power plane within the ellipse represent the input power for a specific complex cross spectral density between the outputs. One phase angle of the output cross spectral density will represent the lowest part of the ellipse on the power plane. This is the minimum power. Add pi radians to establish the point of maximum power. Because in Eq. (11) $P$ is linear in terms of the response cross spectral density matrix, the case for more than two inputs can be interpreted as a series of plots similar to Fig. 2. Each figure represents one of the terms in the summation. The total power is the sum of the individual terms.

\subsection{Summary the solution given the auto-spectra of the outputs}

The solution given the auto-spectra of the inputs is summarized below:

1. Find the phase of the output spectra that will maximize or minimize the trace of the input spectra.

2. Find the cross spectral density matrix of the outputs by combining the derived phase with the given output autospectra for a fully coherent input.

3. Find the input spectral density matrix from $\mathbf{S}_{\mathbf{X X}}=\mathbf{G S}_{\mathbf{Y} \mathbf{Y}} \mathbf{G}^{\prime}$.

\section{Derivation of the minimum/maximum output trace}

In this section we assume the autospectral densities of the inputs are specified and we want to find the input cross spectral densities that minimizes or maximizes the trace of the output autospectral densities. The development is essentially the same as in the previous section. Details are given in Smallwood [13].

$$
P_{y}=\operatorname{trace}\left(\mathbf{S}_{\mathbf{Y Y}}\right)
$$




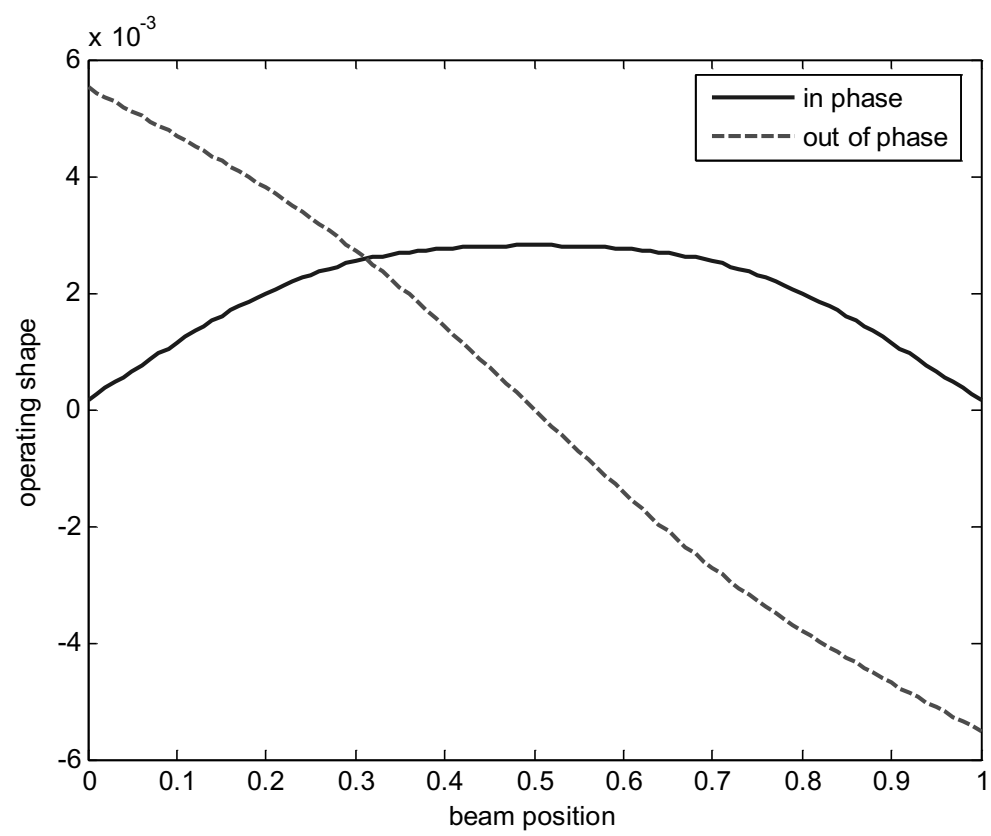

Fig. 16. Example 3, Operating shape near $147 \mathrm{~Hz}$.

Using Eq. (3)

$$
\begin{aligned}
& P_{y}=\operatorname{trace}\left(\mathbf{H S}_{\mathbf{X X}} \mathbf{H}^{\prime}\right) \\
& {\underset{\mathrm{F}}{\mathbf{H}}}_{\mathbf{O}}=\mathbf{H}^{\prime} \mathbf{H} \\
& { }_{H} F_{m j}=\sum_{k=1}^{K} H_{k m}^{*} H_{k j} \quad m=1 \cdots J, \quad j=1 \cdots J
\end{aligned}
$$

As in the previous section let

$$
\begin{aligned}
& X X S_{m j}={ }_{X X} \bar{S}_{m j} \exp \left(i a_{m j}\right) \\
& { }_{H} F_{m j}={ }_{H} \bar{F}_{m j} \exp \left(i b_{m j}\right)
\end{aligned} m=1 \cdots J, \quad j=1 \cdots J
$$

The minimum trace of the output spectral density matrix is given by the input spectral density matrix where

$$
\begin{aligned}
& a_{m j}=\pi+b_{m j} \\
& { }_{X X} \widehat{S}_{m j}={ }_{X X} \bar{S}_{m j} \exp \left(i\left(b_{m j}+\pi\right)\right)
\end{aligned}
$$

The maximum occurs when

$$
a_{m j}=b_{m j}
$$

\subsection{Summary given the auto-spectra of the inputs}

The solution given the auto-spectra of the outputs is summarized below:

1. Find the phase of the input spectra that will maximize or minimize the trace of the output spectra.

2. Find the cross spectral density matrix of the inputs by combining the derived phase with the given input autospectra for a fully coherent input.

3. Find the output spectral density matrix from $\mathbf{S}_{\mathbf{Y Y}}=\mathbf{H S}_{\mathbf{X X}} \mathbf{H}^{\prime}$. 


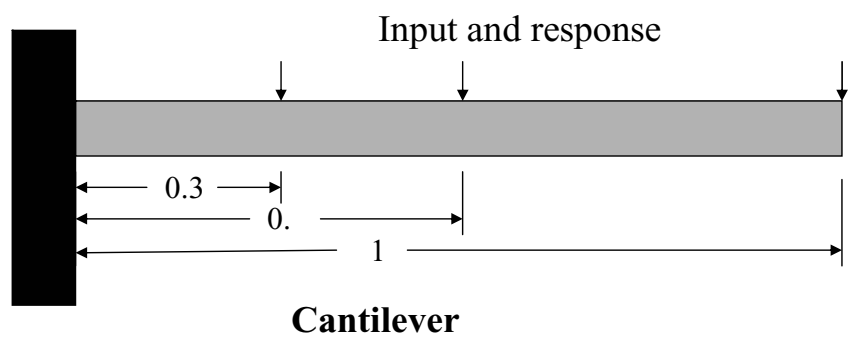

Fig. 17. Sketch of a cantilever beam used in an example.

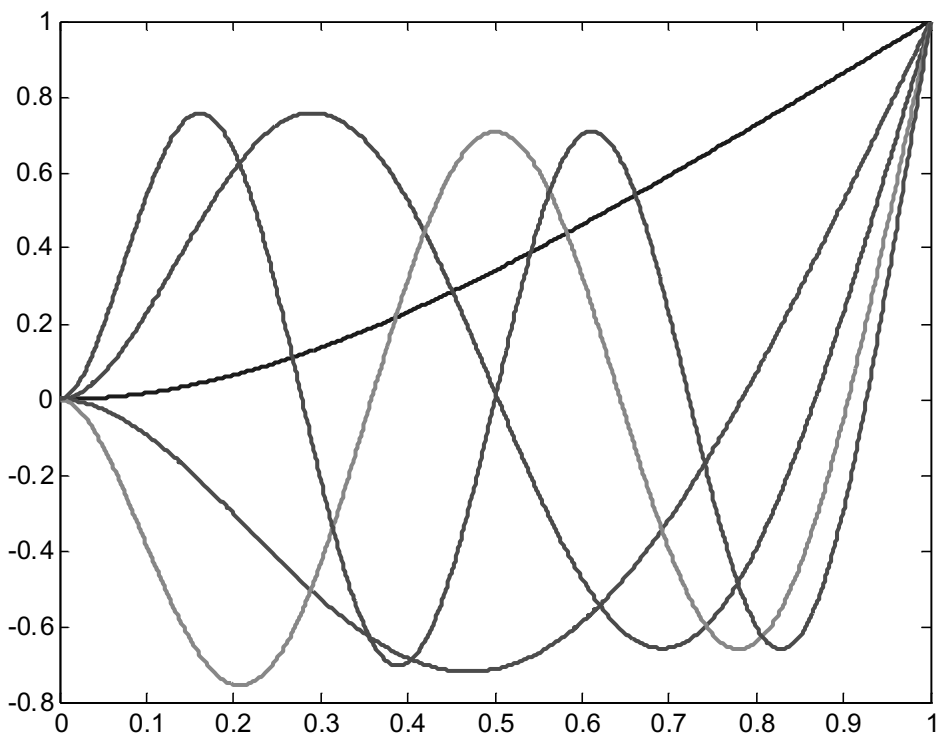

Fig. 18. First 5 mode shapes of the cantilever beam.

\section{Application to transient and sine data}

If the input is a deterministic transient or steady state sine the response can be written in the frequency domain as

$$
\mathbf{Y}=\mathbf{H X}
$$

$\mathbf{Y}$ is now a complex quantity that gives the amplitude and phase of the outputs. $\mathbf{H}$ is the same as before. $\mathbf{X}$ is a complex quantity giving the amplitude and phase of the inputs. For a transient $\mathbf{X}$ and $\mathbf{Y}$ are the Fourier transforms of the transient time histories. For a sine the phase is relative to some arbitrary reference. Some matrix spectral relations are analogous to the definition of cross spectral density (Eq. 2).

$$
\begin{aligned}
& \mathbf{S}_{\mathbf{X X}}=\mathbf{X X ^ { \prime }} \\
& \mathbf{S}_{\mathbf{Y Y}}=\mathbf{Y Y}^{\prime}
\end{aligned}
$$

These equations do not require the use of the expectation operator because the functions are deterministic. The diagonal terms of the spectral matrix are the amplitudes squared of the sine (or Fourier) components. The off diagonal terms define the phase relationships between pairs of signals. Equations (9) and (26) still apply. The development for the deterministic case is the same as for the random waveforms. When the output amplitudes are specified Eqs (21-25) define the relative phase between the outputs. When the input spectral amplitudes are specified, Eqs (31-33) define the relative phase between the inputs. The phase of one of the inputs or outputs must still be defined. If the inputs are specified the outputs are determined from Eq. (3). If the outputs are specified the inputs are determined from Eq. (9). 

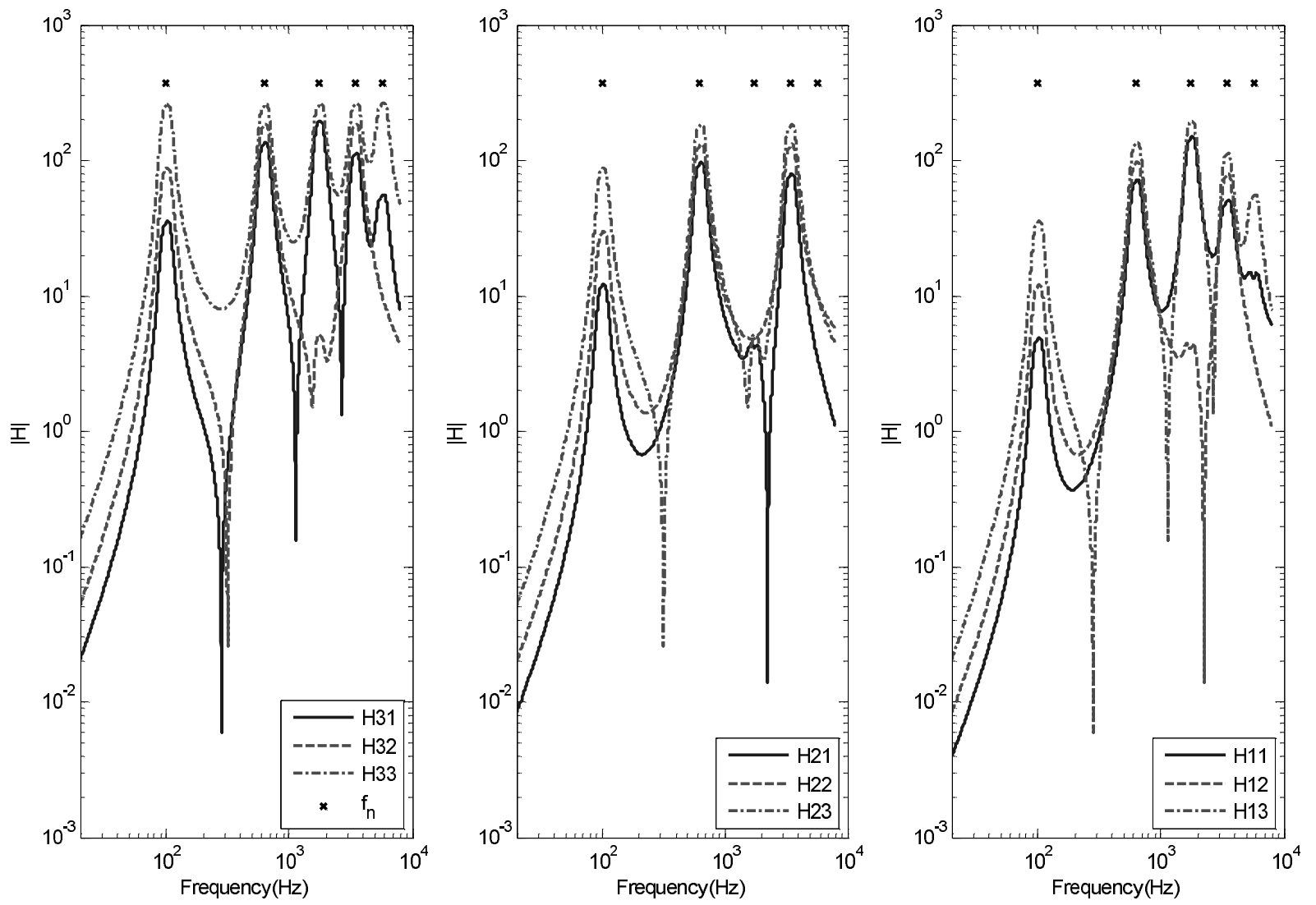

Fig. 19. Frequency response function of the cantilever beam at the driving and response points.

\subsection{Least favorable transient response}

If the system is a single input single output (SISO) system, an input transient that forces all the output frequency domain components to have zero phase results in the least favorable response [3,8]. This is the largest possible peak output for any input with a spectral envelope equal to or less than a specified envelope. Other authors $[1,4,7,14]$ call this the critical response. The maximum response will occur at time zero. In summary the least favorable response is given by

$$
y_{\max }(t)=y(0)=\int_{-\infty}^{\infty}\left|X(f)\left\|H(f)\left|e^{i(-\beta(f)+\beta(f))} d f=\int_{-\infty}^{\infty}\right| X(f)\right\| H(f)\right| d f
$$

where

$$
H=|H| e^{i \beta(f)}
$$

and

$X(f)$ the Fourier transform of the input time history, $x(t)$, and $|X(f)|$ is the magnitude of the input Fourier spectrum amplitude.

If the system is a multiple input single output (MISO) system the results derived in the previous section are a generalization of the least favorable response.

Consider the contribution of an input $X_{1}$ to the output. Just as for the single input least favorable response set

$$
X_{1}(f)=\left|X_{1}\right| e^{i\left(-\beta_{1}(f)\right)}
$$



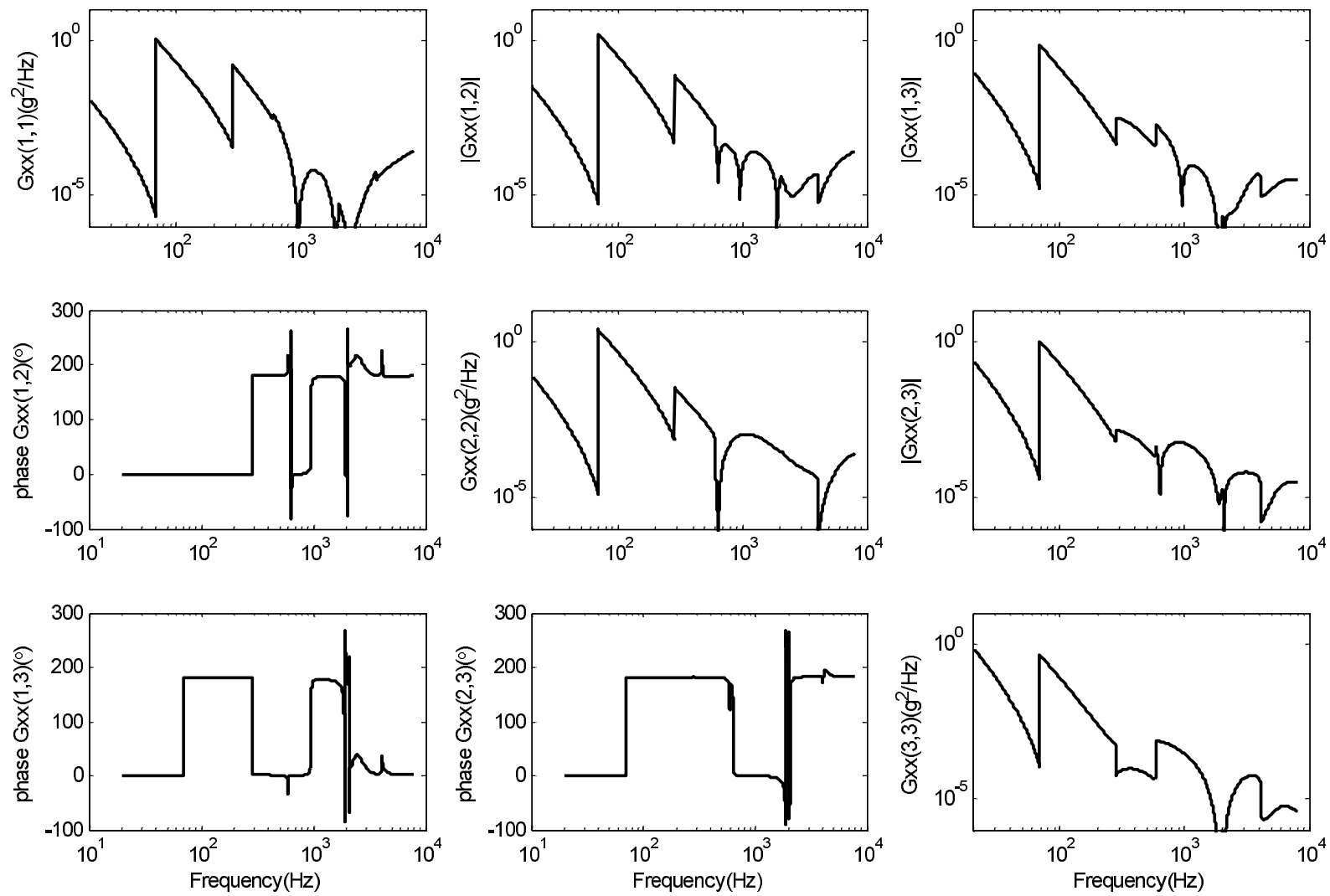

Fig. 20. Input cross spectral density matrix for the cantilever beam example.

where $H_{i}(f)=\left|H_{i}(f)\right| e^{i \beta_{i}(f)}$

which results in

$$
Y_{1}(f)=\left|X_{1}(f)\right|\left|H_{1}(f)\right|
$$

The contribution of $x_{1}(t)$ to the total response will be the same as given by Eq. (37). The relative phases between the inputs are given by $\mathbf{F}_{H}$. For the single output case, the relative phase between $X_{n}$ and $X_{1}$ is given by $\left(-\beta_{n}+\beta_{1}\right)$. The absolute phase of the $X_{n}$ input is then given by $\left(-\beta_{n}+\beta_{1}-\beta_{1}=-\beta_{n}\right)$.

This results in the contribution from the $X_{n}$ input to the output as

$$
Y_{n}(f)=\left|H_{n}\right| e^{i \beta_{n}(f)}\left|X_{n}\right| e^{i\left(-\beta_{n}(f)\right.}=\left|H_{n}\right|\left|X_{n}\right|
$$

The responses caused by the various inputs are all maximum and positive at $t=0$. Therefore, superposition will occur and the total response will be the maximum possible response, the least favorable response generalized for the MISO case.

For the transient case with a single output the minimization of the Fourier magnitude of the output (Section 2) does not seem to be particularly useful as a limiting case.

If the system has multiple inputs and outputs the phase of one of the inputs or outputs must be specified. A single set of inputs that maximizes the response of all the outputs does not exist. If the phase of one of the inputs is known, a single set of inputs that maximizes the trace of the output spectra does exist. If the phase of one of the outputs can be specified (as for example zero phase that results in maximizing the response of that output at $t=0$ ) a set of inputs can be found that produces that response and also maximizes the trace of the spectra of all the responses. 

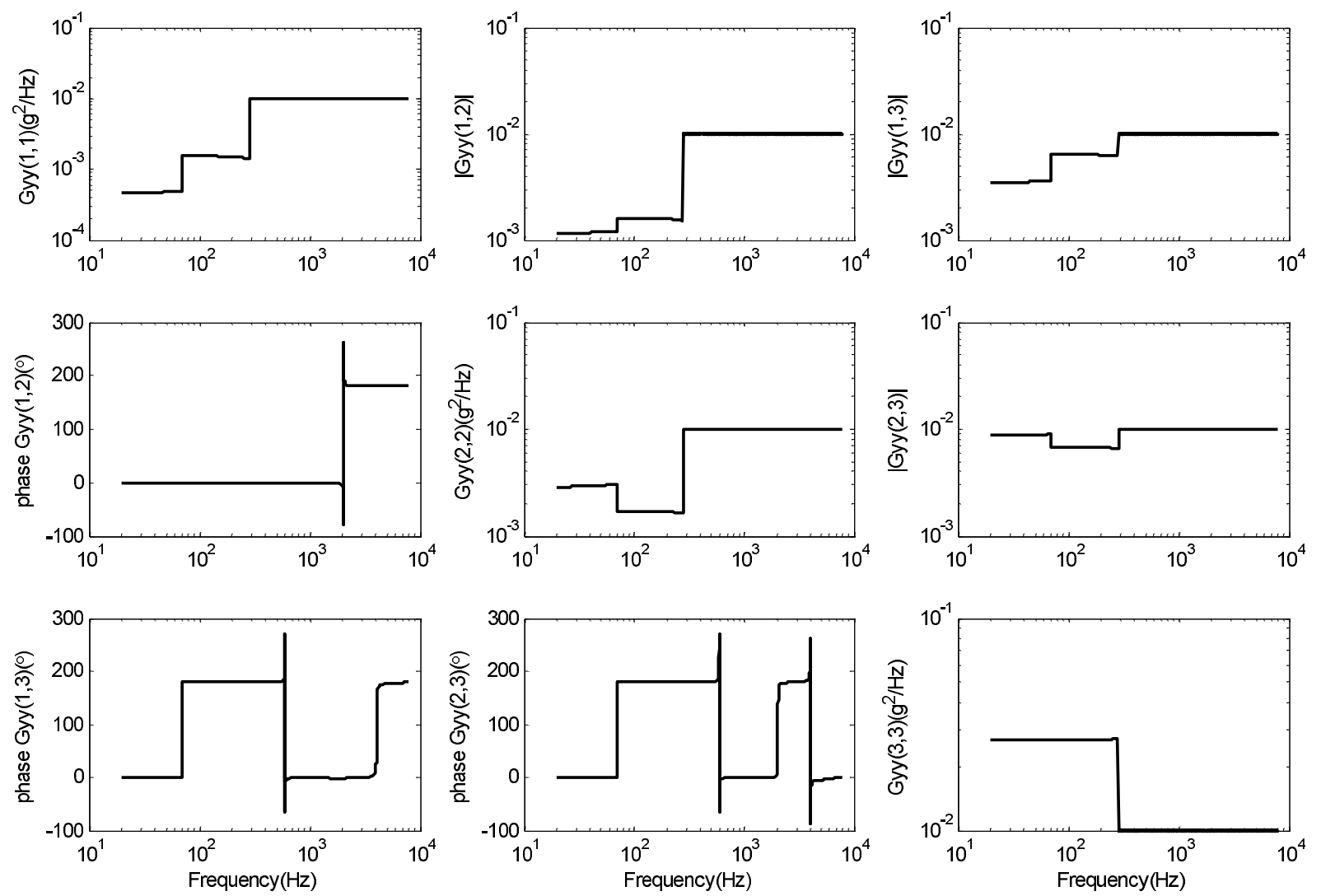

Fig. 21. Output cross spectral density matrix for the cantilever beam example.

\subsection{Pseudo random input}

Whenever we seek to define a pseudo-random input, the phase of the first input can be randomized. If we want to define a pseudo-random signal with a specified temporal modulated envelope, we can generate a pseudo-random signal, transform to the time domain, multiply by a suitable temporal window, and transform back to the frequency domain. This is frequently referred to as a product model. The phases of the remaining inputs are then defined relative to the first windowed signal.

\subsection{A deterministic time history for the first input or output}

The method gives only the relative phase between components. We must arbitrarily choose the phase of one input. For example, we can define the first response (or input) as a specified time history. The FFT of the time history will define the amplitude and phase of the first input (or response) time history. The Fourier magnitude of the remaining inputs (or responses) can be defined independently or can be a defined as a function of the Fourier magnitude of the first signal. The algorithm to minimize the input (or output) defines amplitude and phase of the inputs (or responses) and the phase of the remaining responses (or inputs).

\section{The special cases of multiple inputs and a single output}

If there is only one output the problem reduces to the superposition of the response to $J$ inputs. If the maximum or minimum response is desired the inputs will be fully correlated. If the autospectral densities of the inputs are 


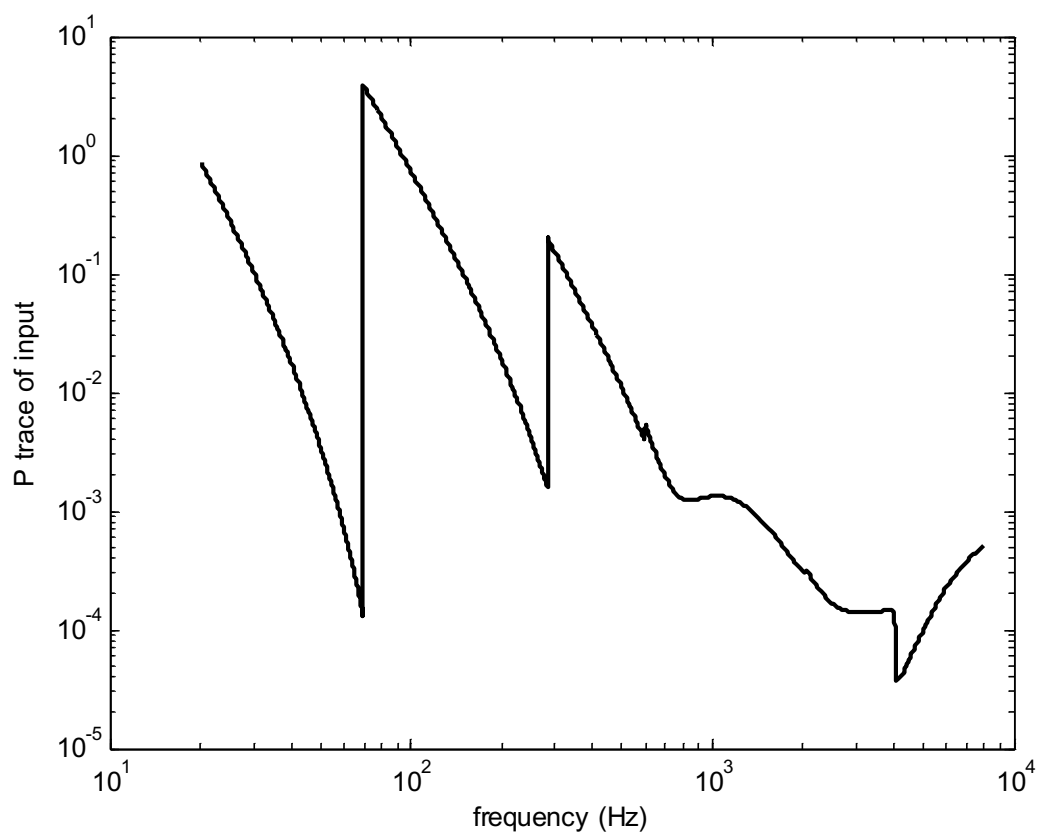

Fig. 22. Trace of the input spectra for the cantilever beam example.

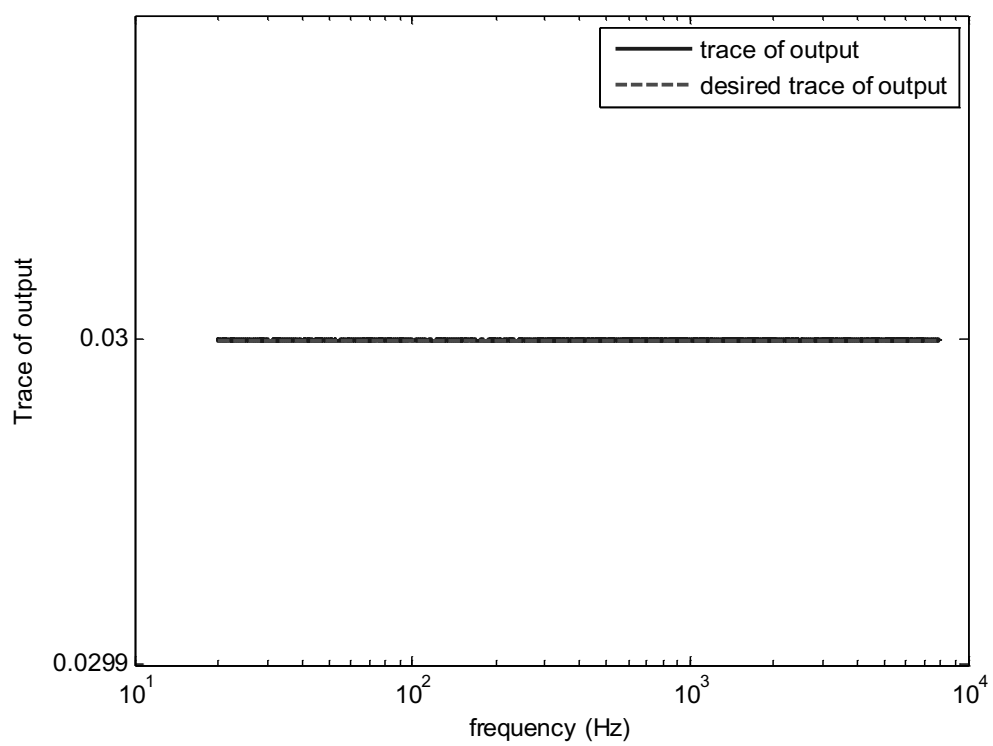

Fig. 23. Trace of the output spectra for the cantilever beam example.

specified, Section 3 is used to determine the phase angles between the inputs for the minimum and maximum output spectral density. If the output auto spectrum is specified, Section 2, Eq. (26) is used to derive the inputs to minimize or maximize the trace of the input spectral density matrix.

If the input is a transient, Section 4.1 has already discussed the solution as a generalization of least favorable response. 

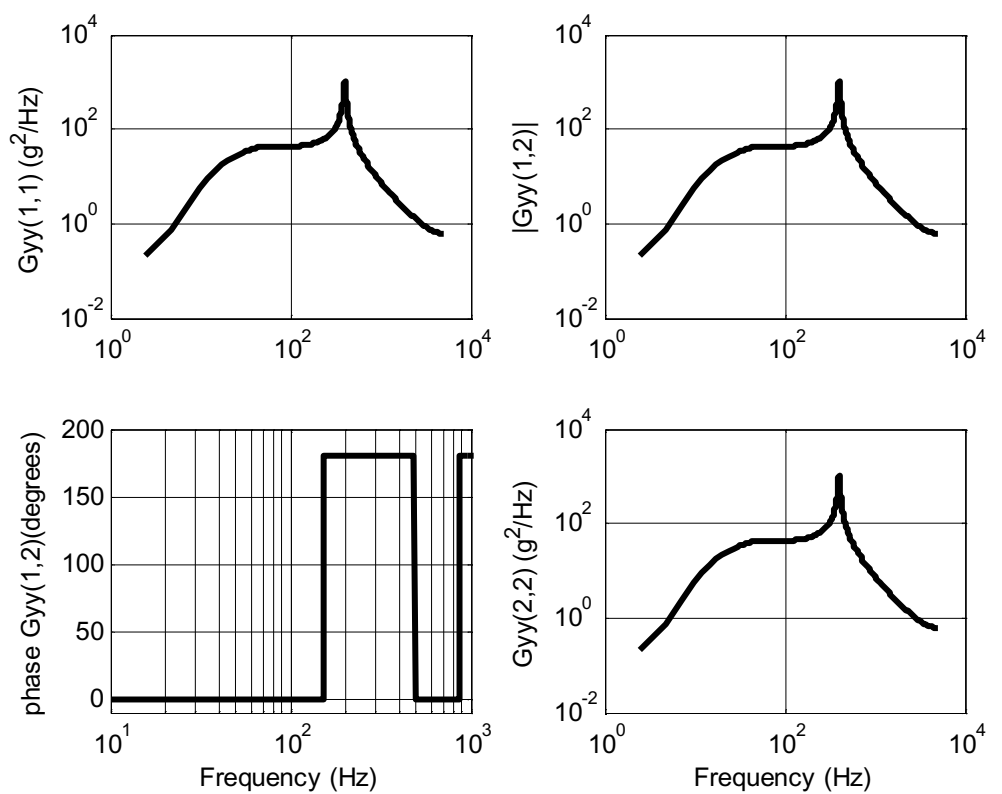

Fig. 24. Example 4, Magnitude of the Fourier spectra of the outputs, scaled as power spectra.
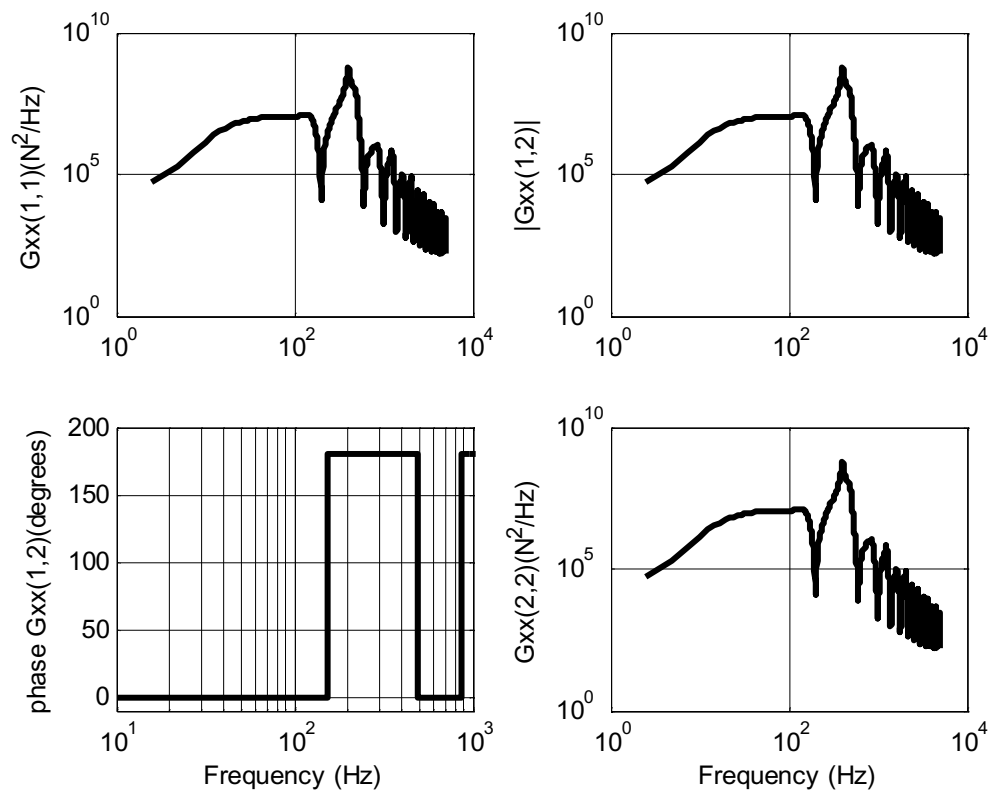

Fig. 25. Example 4, Magnitude of the Fourier spectra of the inputs, scaled as power spectra.

\subsection{Von Mises stress as the output variable}

Consider the von Mises stress that is a quadratic combination of six stress components

$$
\sigma=\left[\begin{array}{lll}
\sigma_{x} \sigma_{y} \sigma_{z} & \sigma_{x y} \sigma_{x z} \sigma_{y z}
\end{array}\right]^{T}
$$

For the square of the von Mises stress the quadratic combination is

$$
p(t)^{2}=\sigma_{x}^{2}+\sigma_{y}^{2}+\sigma_{z}^{2}-\left(\sigma_{x} \sigma_{y}+\sigma_{x} \sigma_{z}+\sigma_{y} \sigma_{z}\right)+3\left(\sigma_{x y}^{2}+\sigma_{y z}^{2}+\sigma_{x z}^{2}\right)
$$




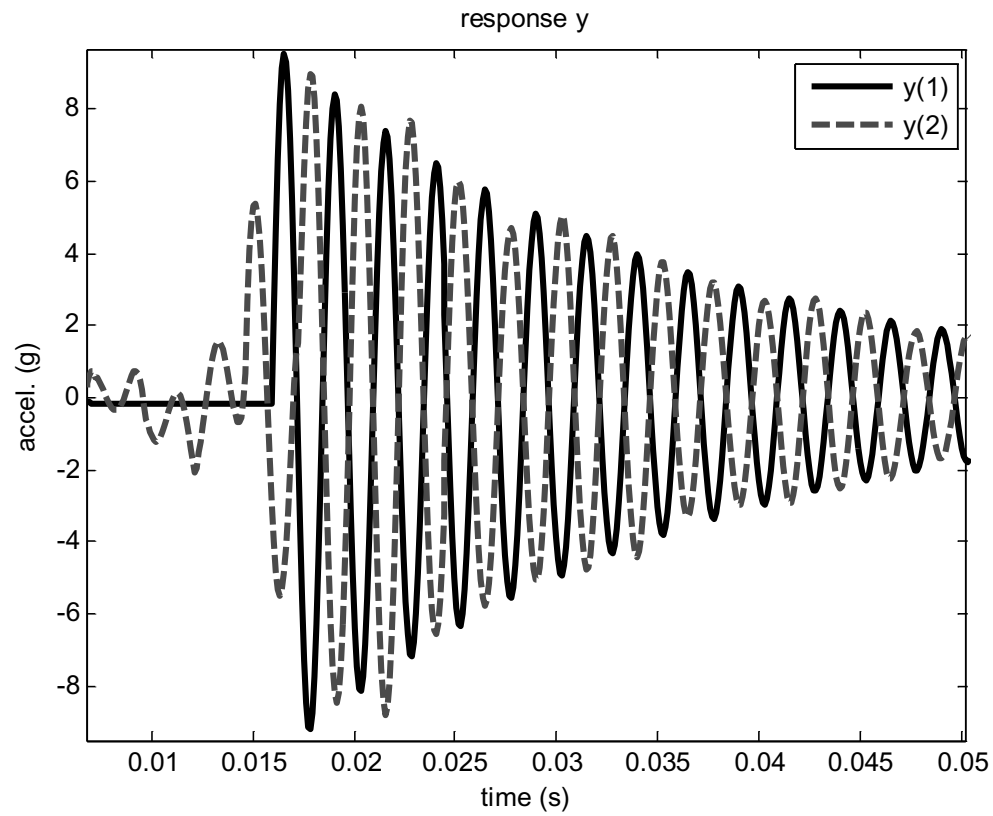

Fig. 26. Example 4, Time histories of the outputs.

Consider quadratic functions of stress, written in the following form

$$
p^{2}=\sigma^{\prime} \mathbf{A} \sigma
$$

For von Mises stress, $\mathbf{A}$ is [7]

$$
\mathbf{A}=\left[\begin{array}{cccccc}
1 & -1 / 2 & -1 / 2 & 0 & 0 & 0 \\
-1 / 2 & 1 & -1 / 2 & 0 & 0 & 0 \\
-1 / 2 & -1 / 2 & 1 & 0 & 0 & 0 \\
0 & 0 & 0 & 3 & 0 & 0 \\
0 & 0 & 0 & 0 & 3 & 0 \\
0 & 0 & 0 & 0 & 0 & 3
\end{array}\right]
$$

The matrix A can be factored using a modified Cholesky decomposition [11] into

$$
\mathbf{A}=\mathbf{B}^{\prime} \mathbf{B}
$$

$$
\mathbf{B}=\left[\begin{array}{cccccc}
1 & -1 / 2 & -1 / 2 & 0 & 0 & 0 \\
0 & \sqrt{3} / 2 & -\sqrt{3} / 2 & 0 & 0 & 0 \\
0 & 0 & 0 & 0 & 0 & 0 \\
0 & 0 & 0 & \sqrt{3} & 0 & 0 \\
0 & 0 & 0 & 0 & \sqrt{3} & 0 \\
0 & 0 & 0 & 0 & 0 & \sqrt{3}
\end{array}\right]
$$

$\mathbf{B}$ has a row of zeros because $\mathbf{A}$ is singular and has a rank of 5.

Let

$$
\mathbf{q}=\mathbf{B} \sigma
$$

Then

$$
p^{2}=\mathbf{q}^{\prime} \mathbf{q}=\sigma^{\prime} \mathbf{B}^{\prime} \mathbf{B} \sigma=\sigma^{\prime} \mathbf{A} \sigma
$$

Because $\mathbf{B}$ is not a function of time, but just a matrix of real constants, transforming to the frequency domain gives 


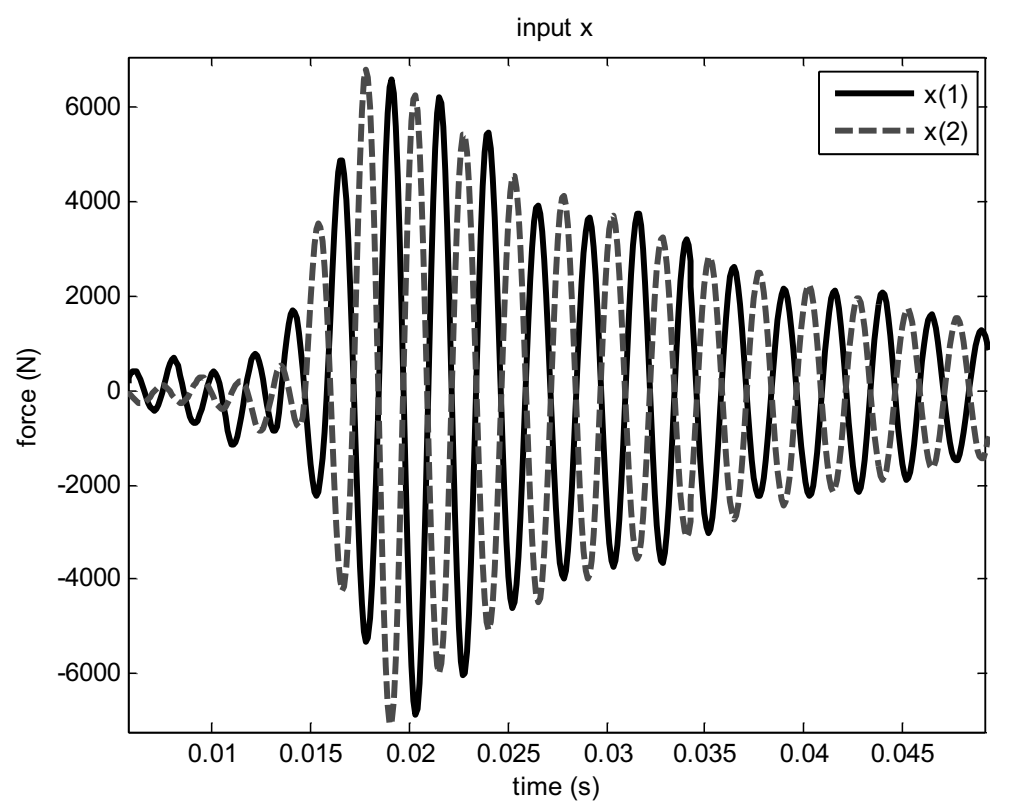

Fig. 27. Example 4, Time histories of the inputs.

$$
\mathbf{Q}=\mathbf{B S}
$$

$$
\mathbf{S}=\mathbf{H X}
$$

which gives

$$
\mathbf{Q}=\mathbf{B H X}
$$

where $\mathbf{Q}$ is the Fourier transform of $\mathbf{q}$, and $\mathbf{S}$ is the Fourier transform of $\sigma$. The stress response is a linear function of the excitation.

$$
P^{2}=\mathbf{Q}^{\prime} \mathbf{Q}=\sum_{j=1}^{6} Q_{j}^{*} Q_{j}
$$

where $P^{2}$ is a spectral measure of the von Mises stress.

Consider the multiple input multiple output (MIMO) system, $\mathbf{Q Q}^{\prime}$. Noting that for any column vector $\mathbf{Q}$, $\mathbf{Q}^{\prime} \mathbf{Q}=\operatorname{trace}\left(\mathbf{Q Q}^{\prime}\right)$, the trace of this system is the same as the above equation

$$
P^{2}=\operatorname{trace}\left(\mathbf{Q} \mathbf{Q}^{\prime}\right)=\sum_{j=1}^{6} Q_{j} Q_{j}^{*}
$$

Thus $\mathbf{Q}$ defines a system with six responses and $J$ inputs that is equivalent to the statement of the von Mises stress.

Combining $\mathbf{B}$ and $\mathbf{H}$ reduces the problem to the MIMO problem already studied. For random vibration, the spectrum of the von Mises stress can be found for inputs with any cross spectral density matrix. If only the input autospectral densities are given, cross spectral densities of the inputs can be found that will minimize or maximize the root-mean-square (rms) von Mises stress. Once the cross spectral density matrix of the inputs is known the individual stress components can be derived from Eq. (3),

$$
\mathbf{S}_{\mathbf{S S}}=\mathbf{H S}_{\mathbf{X X}} \mathbf{H}^{\prime} .
$$




\subsection{Multiple axis vibration tests}

An issue of interest in random vibration or sine testing is the equivalence or severity of a multiple axis vibration test (all three axes excited simultaneously) relative to three single axis tests. The response to each of the three single axis tests can be found from a standard single input single (or multiple) output analysis. The response to the multiple axis test can be handled as a three input single (or multiple) output system. Standard methods allow the determination of the response to any specified input cross spectral density matrix. The methods in this paper also allow the determination of the specific input cross spectral densities that will result in the maximum and minimum spectrum of the response (or the trace of the output spectra for the multiple output case). To determine the severity of the tests a damage criteria must be used which relates the combination of response spectra and duration of the test to damage. If such a model exists the tests can be compared.

\section{Examples of stationary random excitation}

The examples shown are relatively simple to illustrate the concept. The simplicity aids our intuition about the validity of the solutions. More complicated solutions could be shown, but are difficult to interpret. It is expected that the most common applications will include MIMO testing and models with multiple force inputs. Therefore, the free-free boundary conditions are emphasized. To illustrate the method a simple free-free bar is excited axially for examples 1, 2, and 4. The bar has its first resonance at $100 \mathrm{~Hz}$. The damping is set at $1 \%$ for all modes. For the first example the bar will be excited at the two ends (designated as 0 and 1, see Fig. 3). The response will be measured at the same locations. For the second example three inputs will be used at 0.6 and 1 times the length of the bar. The response will be measured at the two ends $(0$ and 1 . This bar could represent a slip table excited at various points. The 3 by 3 matrix of frequency response functions $(\mathrm{g} / \mathrm{N})$ is plotted at Figs 4 (magnitude) and 5 (phase). Reciprocity holds for this system, therefore the frequency response function matrix is symmetric.

\subsection{Example 1 Two inputs - two outputs}

The desired response is a flat spectrum of $0.01 \mathrm{~g}^{2} / \mathrm{Hz}$ from 10 to $1000 \mathrm{~Hz}$ at location 0 and $0.011 \mathrm{~g}^{2} / \mathrm{Hz}$ from 10 to $1000 \mathrm{~Hz}$ at location 1. The desired coherence is near zero. The bar is rigid at very low frequencies and we would expect control to be difficult as the frequency decreases because the desired low coherence. The frequency response function matrix, $\mathrm{H}$, is essentially singular at very low frequencies. This was accommodated in the inverse of $\mathrm{H}$ using the condition number of the matrix. If the condition was below a specified threshold one of the singular values was set to zero. The results are shown in Figs 6-8. The input cross spectral density matrix is shown as Fig. 6 . The magnitude of the cross spectrum is in the upper right, the phase of the cross spectrum in the upper right is shown in the lower left. Notice the rapid increase in the inputs as the frequency decreases, until the matrix is declared singular at about $25 \mathrm{~Hz}$. Below this frequency the coherence is near unity, not zero as required. The physical constraints require the high coherence with a reasonable force input. The response spectral density is shown as Fig. 7. Notice that the desired spectra amplitude or coherence is not maintained below $25 \mathrm{~Hz}$. A compromise is achieved within the constraints of the system. The desired response is achieved about above $25 \mathrm{~Hz}$. The trace of the input spectra is shown as Fig. 8.

\subsection{Example 2 Three inputs - two outputs}

The same bar as in example 1 will be used. Three inputs will be used at 0, 0.6 and 1 times the length of the bar. The outputs will still be a 0 and 1 . The requirement will be $0.01 \mathrm{~g}^{2} / \mathrm{Hz}$ from 10 to $1000 \mathrm{~Hz}$ at location 0 and at location 1 . The phase will not be specified. The objective is to pick a phase that will minimize the trace of the input spectral density matrix. The minimum input spectral density matrix is shown as Fig. 9 as a solid line. As before the amplitude of the cross spectra is shown in the upper right and the phase is shown in the lower left. The resulting output spectral density matrix is shown as Fig. 10 as a solid line. The trace of the minimum input is shown as Fig. 11 as a solid line. The response that maximizes the trace of the inputs will also be computed. These are shown as dashed lines in Figs 9-11. All other phase and coherence relationships between the responses will be between these extremes. Notice that each individual input is not maximum for the maximum condition, the trace is maximum. An example can be seen near $200 \mathrm{~Hz}$. 


\subsection{Example 3, A beam driven transversely at both ends}

In this example a free-free beam is excited in the transverse (lateral) direction. The beam is of unit length. The first free-free bending response is at $300 \mathrm{~Hz}$. The input is force at the two extreme ends ( $x=0$ and 1 , see Fig. 2). The response is acceleration at the same locations. This system has two rigid body modes at zero frequency (translation and rotation). With two inputs and two responses the matrix of frequency response functions should not be singular at any frequency. The modal damping is set at $1 \%$. The two by two matrix of frequency response functions is shown as Fig. 12. The output auto spectral densities are specified as $0.01 \mathrm{~g}^{2} / \mathrm{Hz}$ from 100 to $1000 \mathrm{~Hz}$. The minimum and maximum input auto spectral densities to achieve this level were calculated. The input and output spectral density matrices are shown in Figs 13 and 14. The output spectra are at the desired level at all frequencies within the round off error of the calculations. The trace of the input spectral density matrix $(\mathrm{P})$ is shown as Fig. 15. There are several items of interest in this example. First, the rigid body rotation mode is easier to excite than the rigid body translation at low frequencies, therefore the minimum input results in the input forces with a phase angle of $180^{\circ}$ below about $220 \mathrm{~Hz}$. The maximum input at low frequencies occurs with the input forces in phase. Note the input required is minimum at the free-free resonances of the beam as expected. The phase of the inputs is always near $0^{\circ}$ or $180^{\circ}$ with a fast transition between the two values. This is because of the low damping. The large peak in the maximum input (the input to keep the responses in phase) near $147 \mathrm{~Hz}$ deserves an explanation. Figure 16 plots the operating shape at this frequency when the inputs are in and out of phase. When the inputs are out of phase the shape is primarily rigid body rotation with a little of the first bending mode is excited. The rigid body translation is suppressed. When the inputs are in phase the shape is like the first bending mode of a pinned-pinned beam. Forcing the responses to be in phase has the effect of imposing a pinned-pinned boundary condition on the beam. The input source must look like large impedance to the beam to enforce this condition.

Other input conditions, (other phase angle and/or coherence of less than one) will result in the trace of the inputs between the two extremes of minimum and maximum conditions. The input trace is bounded between these two extremes.

The input auto spectral densities could also be specified and the extremes of the response auto spectra densities calculated. These extremes bound the responses for any inputs with the specified auto spectral densities.

\section{Example 4, Cantilever beam, fixed at the zero end, excited and measured at location $0.3,0.5$, and the free end, 1.0}

To illustrate the method for boundary condition other than free-free, a cantilever beam of unity length will be used. Figure 17 is a sketch of the beam. The first five modal frequencies of the beam will be used in the analysis. The mode shapes are shown in Fig. 18. The frequency response functions for the three excitation and response points are shown in Fig. 19. The natural frequencies are shown as x's on the top of the figure. The damping is $1 / 2$ $\%$ of critical at all five modes. The desired response was 0.01 at all three response locations. This system does not have a rigid body mode. At low frequencies only the first mode is easily excited. Therefore, it would be very difficult to excite all three response locations at the desired amplitude. A very large drive force would be required to achieve the desired response at all three response points at low frequencies. To avoid this, singular values below a threshold were set to zero. The matrix, F (as defined in Eq. 15), is has one singular value (above the threshold) up to about $72 \mathrm{~Hz}$, two singular values between 72 and $300 \mathrm{~Hz}$ and three singular values above $300 \mathrm{~Hz}$. This limits the magnitude of the input trace. The trace of the outputs is as required, even though the individual responses are not as desired. Figure 20 shows the resulting input cross spectral density. The magnitudes are shown in the upper half of the figure and the phase is shown in the lower half of the figure. The cross spectral densities of the outputs are shown as Fig. 21. The trace of the input is shown as Fig. 22. The trace of the output is shown as Fig. 23. Note the discontinuities in the trace of the input (near 72 and $300 \mathrm{~Hz}$ ) as additional singular values are computed above the tolerance were achieved. The desired response is achieved for frequencies above $300 \mathrm{~Hz}$ as more modes are easily excited. 


\section{Example 5, Bar, Inputs at points 0 and 1, response locations at 0 and 1, Response 1 is a decaying exponential at $400 \mathrm{~Hz}$}

This example will illustrate how the technique can be used in a transient case. The input will again be at points 0 and 1 on a bar. The desired response at point one (solid trace, Fig. 26) is defined as an exponentially decaying sinusoid at a $400 \mathrm{~Hz}$, where a compensating waveform at $10 \mathrm{~Hz}$ has been added to force the initial and final acceleration, velocity, and displacement to zero. The decay rate of the $400 \mathrm{~Hz}$ component is $2 \%$. The decay rate of the compensating waveform is 1 . This waveform has most of its energy in a band of frequencies near $400 \mathrm{~Hz}$. The second response is defined as having the same Fourier magnitude as the first response with the phase unspecified. Near $400 \mathrm{~Hz}$ the system is dominated by the first mode. Therefore, for a minimum input trace we would expect the inputs be nearly out phase. We expect the response at point 2 to be similar to the response at point 1 but inverted. Figure 24 is the as the Fourier spectra (scaled as power spectra) of the outputs for a minimum input trace. The first output (upper left plot) has the typical shape of an exponentially decaying sinusoid with the very low frequency content removed. The upper right plot is the cross spectrum amplitude between the two outputs. The lower left is the relative phase between the two outputs. The lower right is the required spectral amplitude of the second output (it is the same as the spectrum of the first input). The spectra of the inputs are shown as Fig. 25. The resulting output time histories are shown as Fig. 26. The resulting input time histories are shown as Fig. 27. Note that even though the phase of the second output was set to minimize the trace of the input spectra, the time history still resembles an exponentially decaying sinusoid. The two inputs and two outputs are nearly out of phase as expected.

Several more examples are given in Smallwood [13].

\section{Future work}

This work was suggested by a practical problem which has not been completed. An aerospace subsystem is supported at essentially four points. A finite element model of the subsystem was being constructed. This results in a natural input of 3 triaxial forces at each of the 4 support points for a total of 12 input forces. The rotations at the supports were ignored. The model was to be validated with a modal test. The intent was to predict component responses within the model to flight environments. The problem was that the input forces are unknown. All that is known from flight data is the acceleration response at two triaxial accelerometers and one single axis accelerometer on three components within the subsystem, for a total of 7 measurements. Only the autospectra are known at these measurement locations. The problem was to derive a set of force inputs to the model that would duplicate the measured responses. The forced response of the model would then be used to predict the motion at other points. Obviously the solution is not unique. A reasonable solution was sought which led to the present work.

Experimental verification of this work still needs to be performed.

\section{Conclusions}

A systematic procedure has been derived to handling stationary random problems with multiple inputs and multiple outputs where only autospectra are known. The method derives cross spectra that will minimize or maximize the trace of the input or output autospectra. Other inputs will produce inputs or responses between the extremes. The method can also be applied to nonstationary random inputs and to deterministic sine and transient problems.

\section{Acknowledgements}

This work was supported in part by Sandia National Laboratories. Sandia is a multiprogram laboratory operated by Sandia Corporation, a Lockheed Martin Company, for the United States Department of Energy's National Nuclear Security Administration under Contract DE-AC04-94AL85000. 


\section{References}

[1] A. Baratta, I. Elishakoff, G. Zaccaro and M. Shinozuka, A generalization of the Drenick-Shinozuka model for bounds on the seismic response of a single-degree-of-freedom system, Earthquake Engineering \& Structural Dynamics 27 (1998), $423-437$.

[2] J.S. Bendat and A.G. Piersol, Random Data, Analysis and Measurement Procedures, 3rd edition, Wiley, 2000.

[3] R.F. Drenick, Model-Free Design of Aseismic Structures, J. of the Engr. Mech. Div., Proc. of the ASCE (August 1970), $483-493$.

[4] O.A. Lopez, A.K. Chopra and J.J. Hernandez, Critical response of structures to multicomponent earthquake excitation, Earthquake Engineering \& Structural Dynamics 29(12) (Dec. 2000), 1759-1778.

[5] O.A. Lopez, A.K. Chopra and J.J. Hernandez, Evaluation of combination rules for maximum response calculation in multicomponent seismic analysis, Earthquake Engineering \& Structural Dynamics 30(9) (Sep. 2001), 1379-1398.

[6] T.L. Paez, D.O. Smallwood and E.J. Buksa, Random Control at $\mathrm{n}^{2}$ Points Using $\mathrm{n}$ Shakers, Proc. of the IES (May, 1987), 271-275.

[7] D.J. Segalman, C.W.G. Fulcher, G.M. Reese and R.V. Field Jr., An Efficient Method for Calculating RMS von Mises Stress in a Random Environment, Journal of Sound and Vibration 230(2) (17 Feb. 2000), 393-410.

[8] M. Shinozuka, Maximum Structural Response to Seismic Excitations, J. of the Engr. Mech. Div., Proc. of the ASCE (October, 1970), 729-738.

[9] D.O. Smallwood, An Extension of a Transient Vibration Technique Using Least Favorable Response, Sandia National Laboratories Report SC-RR-720735, (Nov. 1972).

[10] D.O. Smallwood, A Transient Vibration Test Technique Using Least Favorable Responses, Shock \& Vibration Bulletin (No. 43), Part 1, June, 1973, 151-164.

[11] D.O. Smallwood and T.L. Paez, A frequency domain method for the generation of partially coherent normal stationary time domain signals, Shock and Vibration 1(1) (1993), 45-53.

[12] D.O. Smallwood, T.D. Woodall and E.J. Buksa, Minimum drive requirements for a multiple input multiple output linear system, Proc. of the Institute of Environmental Sciences (now the Institute of Environmental Sciences and Technology) (1986), 295-302.

[13] D.O. Smallwood, Extreme Inputs/Outputs for Multiple Input Multiple Output Linear Systems,' SAND 2005-5307, Sandia National Laboratories Report, Albuquerque, NM, October 2005.

[14] I. Takewaki, A new method for non-stationary random critical excitation, Earthquake Engineering \& Structural Dynamics 20(4) (April 2001), 519-536.

[15] M.A. Underwood and T. Keller, Applying Coordinate Transformations to Multi Degree of Freedom Shaker Control, Proc. of the 74th Shock and Vibration Symposium, 2003. 

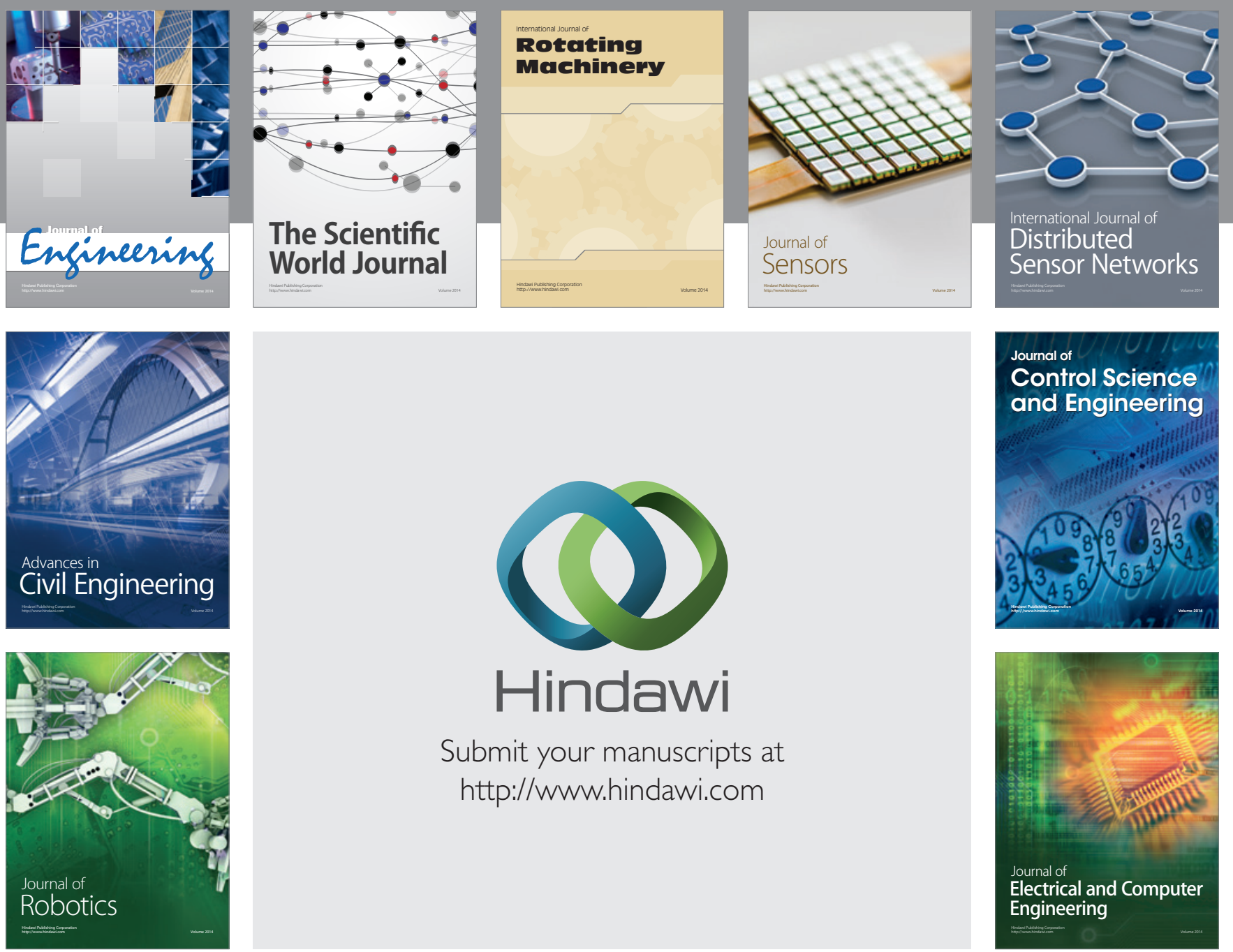

Submit your manuscripts at

http://www.hindawi.com
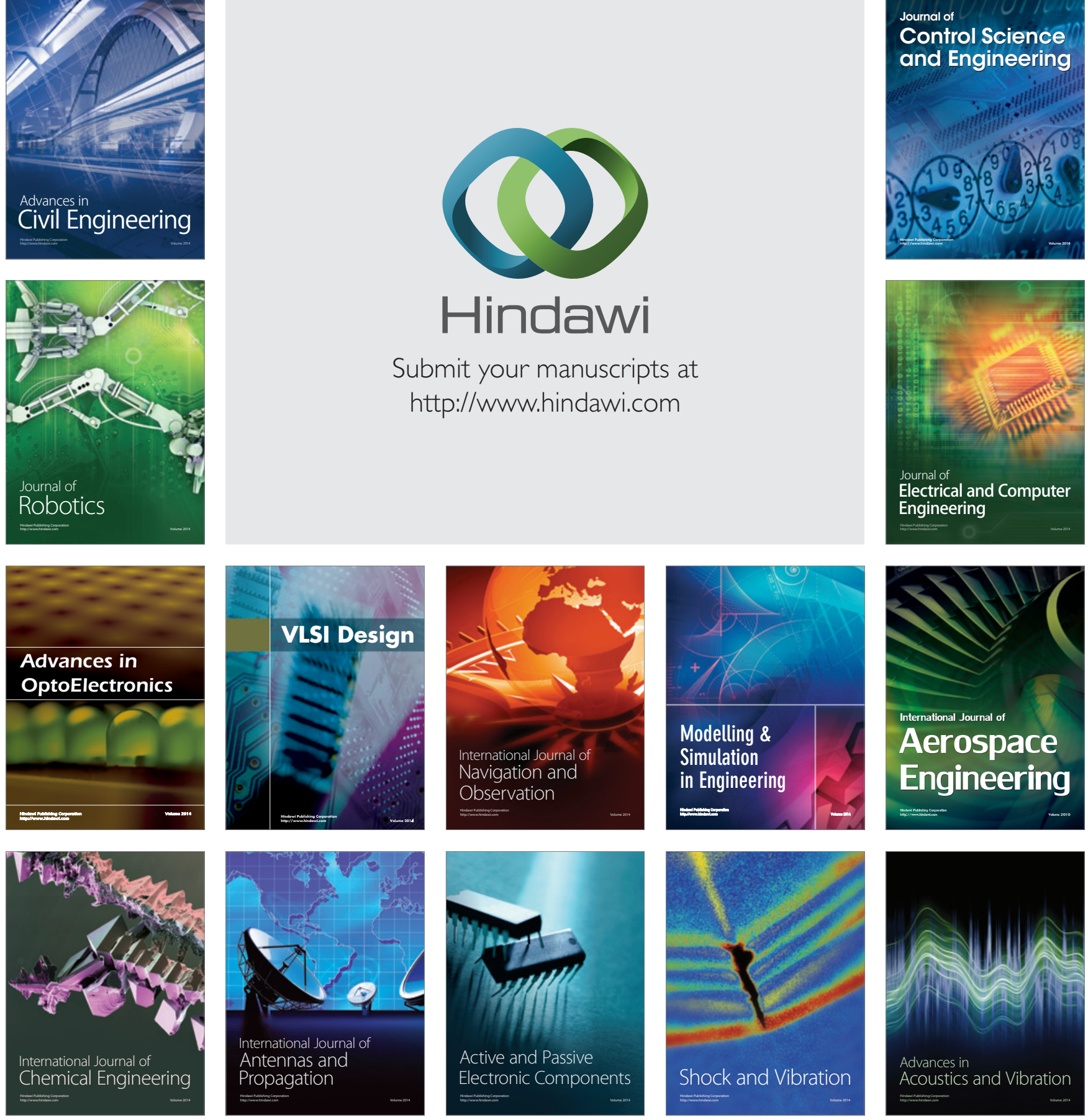